\title{
Exploring LoRa for Long-range Through-wall Sensing
}

\author{
FUSANG ZHANG, Institute of Software, Chinese Academy of Sciences and Peking University, China \\ ZHAOXIN CHANG, KAI NIU, Peking University, China \\ JIE XIONG, University of Massachusetts Amherst, USA \\ BEIHONG JIN, Institute of Software, Chinese Academy of Sciences and University of Chinese Academy of Sciences, China \\ QIN LV, University of Colorado Boulder, USA \\ DAQING ZHANG, Peking University, China and Institut Polytechnique de Paris, France
}

\begin{abstract}
Wireless signals have been extensively utilized for contactless sensing in the past few years. Due to the intrinsic nature of employing the weak target-reflected signal for sensing, the sensing range is limited. For instance, WiFi and RFID can achieve 3-6 meter sensing range while acoustic-based sensing is limited to less than one meter. In this work, we identify exciting sensing opportunities with LoRa, which is the new long-range communication technology designed for IoT communication. We explore the sensing capability of LoRa, both theoretically and experimentally. We develop the sensing model to characterize the relationship between target movement and signal variation, and propose novel techniques to increase LoRa sensing range to over 25 meters for human respiration sensing. We further build a prototype system which is capable of sensing both coarse-grained and fine-grained human activities. Experimental results show that (1) human respiration can still be sensed when the target is 25 meters away from the LoRa devices, and 15 meters away with a wall in between; and (2) human walking (both displacement and direction) can be tracked accurately even when the target is 30 meters away from the LoRa transceiver pair.
\end{abstract}

CCS Concepts: • Human-centered computing $\rightarrow$ Ubiquitous and mobile computing systems and tools.

Additional Key Words and Phrases: Wireless sensing; Long range sensing; LoRa signal; Modeling

ACM Reference Format:

Fusang Zhang, ZhaoXin Chang, Kai Niu, Jie Xiong, Beihong Jin, Qin Lv, and Daqing Zhang. 2020. Exploring LoRa for Long-range Through-wall Sensing. Proc. ACM Interact. Mob. Wearable Ubiquitous Technol. 4, 2, Article 68 (June 2020 ), 27 pages. https://doi.org/10.1145/3397326

Authors' addresses: F. Zhang, State Key Laboratory of Computer Sciences, Institute of Software, Chinese Academy of Sciences; Key Laboratory of High Confidence Software Technologies (Ministry of Education), School of Electronics Engineering and Computer Science, Peking University, Beijing, China; E-mail: zhangfusang@otcaix.iscas.ac.cn. Z. Chang, K. Niu, Key Laboratory of High Confidence Software Technologies (Ministry of Education), School of Electronics Engineering and Computer Science, Peking University, Beijing, China; E-mail:\{czx97, xjtunk\}@pku.edu.cn. J. Xiong, College of Information and Computer Sciences, University of Massachusetts Amherst, USA; E-mail:jxiong@cs.umass.edu. B. Jin, State Key Laboratory of Computer Sciences, Institute of Software, Chinese Academy of Sciences; University of Chinese Academy of Sciences, Beijing, China; E-mail:Beihong@iscas.ac.cn. Q. Lv, Department of Computer Science, University of Colorado Boulder, USA; Email:qin.lv@colorado.edu. D. Zhang, Key Laboratory of High Confidence Software Technologies (Ministryof Education), School of Electronics Engineering and Computer Science, Peking University, Beijing, China, Telecom SudParis, InstitutPolytechnique de Paris, Evry, France, dqzhang@sei.pku.edu.cn. Corresponding Author: Daqing Zhang, Beihong Jin; E-mail:dqzhang@sei.pku.edu.cn, Beihong@iscas.ac.cn.

Permission to make digital or hard copies of all or part of this work for personal or classroom use is granted without fee provided that copies are not made or distributed for profit or commercial advantage and that copies bear this notice and the full citation on the first page. Copyrights for components of this work owned by others than ACM must be honored. Abstracting with credit is permitted. To copy otherwise, or republish, to post on servers or to redistribute to lists, requires prior specific permission and/or a fee. Request permissions from permissions@acm.org.

(C) 2020 Association for Computing Machinery.

$2474-9567 / 2020 / 6-A R T 68 \$ 15.00$

https://doi.org/10.1145/3397326

Proc. ACM Interact. Mob. Wearable Ubiquitous Technol., Vol. 4, No. 2, Article 68. Publication date: June 2020. 


\section{INTRODUCTION}

Wireless sensing has attracted significant amounts of attentions in recent years and various wireless signals have been explored for contactless sensing, including WiFi [12, 53], RFID [54, 56], FMCW radar [11, 13], ultrasound [52, 55], 60GHz radar [25, 39], UWB [51], and visible light [30, 49]. Many new applications have been enabled, such as localization [26], contactless activity sensing [44], respiration monitoring [13], and keystroke detection [14]. While promising in many aspects, one key limitation of current wireless sensing technologies is the short sensing range. Although the communication range of WiFi can reach 20-50 meters indoors, its sensing range is only 3-6 meters due to the weak target-reflected signal used to sense target movement. Furthermore, existing wireless sensing solutions require at least one of the transceivers to be close to the target, which may not be feasible in certain scenarios. Table 1 shows the sensing capabilities of commonly-used wireless technologies in terms of operational range and through-wall sensing capability. We can see that long-range through-wall sensing is still missing with existing wireless sensing technologies. We believe long-range through-wall sensing can be particularly useful in application scenarios such as health and wellbeing monitoring [43], survivor search in rescue scenes [35] [50], and intrusion detection [29]. For example, in a fire scenario, the sensing device can not be placed too close to the building and the long sensing range is critical in this scenario. Another good example is to contactlessly monitor the patients with infectious virus such as COVID-19 through walls.

This work explores exciting new opportunities to significantly increase the sensing range with the introduction of LoRa [4]. LoRa is one of the major low-power wide-area network (LPWAN) technologies that aim to sustain connections among IoT devices over a long distance. LoRa adopts a spread spectrum modulation technique, from which a coding gain is derived as the signal can be received with a very low Signal Noise Ratio (SNR). Meanwhile, lower bandwidth signal of LoRa reduces the impact of adjacent signal interference and has high signal power with this narrower slice of channel. Thus, the transmission range can be up to ten kilometers in rural areas and a few kilometers in urban areas [16] [41]. LoRa devices are low cost (\$5 - 15 per unit [7]) and are being widely deployed in many Asian and European countries. In the U.S., Semtech announced in 2018 that city-wide LoRa networks will be available in 10 cities [10] and it is predicted that the number of connected LoRa nodes will reach 10 billion in the next 5 years [9]. To date, LoRa networks have been used for applications including environment monitoring [33][15], facility management [47], intelligent building control [28], vehicle tracking [34], and smart agriculture [48]. However, existing work only utilizes the data communication function of LoRa to transfer the sensor data collected.

We envision that the LoRa signal itself can be powerful and preferable for long-range contactless sensing. We explore this vision, through both theoretical modelling and experimental studies, to gain a deep understanding of the sensing capabilities of LoRa in terms of sensing range and granularity. Specifically, we aim to answer the following questions:

- How do we model LoRa signal propagation in the air and develop the underlying theories for LoRa-based sensing?

- What is the sensing range of LoRa? Can we improve it via signal processing techniques?

- Is it possible for both LoRa transceivers to be far away from the sensing target which is not feasible with current WiFi or RFID solutions? In a more challenging scenario, is it possible for LoRa to sense a far away target behind multiple walls? What is the sensing range limit of LoRa?

- Can LoRa signals sense both coarse-grained movements such as walking and fine-grained movements such as human respiration from afar?

- How robust is LoRa sensing against interference? How can we address the interference issue? 
Table 1. Comparison of Different Contactless Sensing Technologies and Systems

\begin{tabular}{ccccccc}
\hline & WiFi & RFID & FMCW radar & Ultrasound & $60 \mathrm{GHz}$ radar & Visible light \\
\hline Hardware Cost & Low & Low & High & Low & High & Low \\
Power Consumption & High & Low & High & Low & High & Low \\
\hline Human & IndoTrack & TagTrack & WiTrack & - & MMW system & StarLight \\
tracking system & {$[32]$} & {$[45]$} & {$[11]$} & $\begin{array}{c}\text { Short range } \& \\
\text { high precision } \\
\text { application }\end{array}$ & $\begin{array}{c}7 \mathrm{~m}(2 \times 8 \\
\text { antenna arrays })\end{array}$ & $\begin{array}{c}\text { 20 LED panels } \\
\text { Tracking range }\end{array}$ \\
& $6 \mathrm{~m}$ & $4.8 \mathrm{~m}$ & $9 \mathrm{~m}$ & $\times \mathrm{m}$ \\
Through wall & $\times$ & $\times$ & $\checkmark$ & $\times$ & $\times$ & $\times$ \\
\hline Respiration & Fullbreath & TagBreath & Vital-Radio & C-FMCW & Doppler Radar & $\times$ \\
sensing system & {$[62]$} & {$[56]$} & {$[13]$} & {$[55]$} & {$[25]$} & $\times$ \\
Sensing range & $3.7 \mathrm{~m}$ & $4 \mathrm{~m}$ & $8 \mathrm{~m}$ & $0.8 \mathrm{~m}$ & $2 \mathrm{~m}$ & $\times$ \\
Through wall & $\times$ & $\times$ & $\checkmark(4 \mathrm{~m})$ & $\times$ & $\times$ & $\times$ \\
\hline
\end{tabular}

In this work, we find that chirp-based LoRa signal presents us with great opportunities for long-range sensing. We first develop the LoRa signal propagation model to capture the relationship between target movement and signal variation. Instead of using single-antenna signal, we propose to utilize the ratio of the signals received by two different antennas at the receiver, which has multiple benefits. The first benefit is that the sensing range can be greatly increased with this simple division operation between two signals. The main reason is that when the target is far away, the reflected signal becomes very weak and can be easily buried in noise. This division operation actually cancels out the noise and the obtained signal ratio shows clearer variation patterns corresponding to target movement. Second, since the transmitter and receiver are not time synchronized, the LoRa signal has a random signal phase offset. The proposed division operation can also cancel out the random phase offsets, thus allowing us to employ the stable phase of signal ratio for sensing. We further quantify the change of signal ratio corresponding to target movement for highly-accurate sensing. Specifically, both the distance and direction of target movement can be accurately tracked from far away and through walls.

Besides the theoretical model, which we believe lays a solid foundation in guiding the design of LoRa sensing, we also build the LoRa sensing prototype and systematically study the sensing capability of LoRa signals. We implement two sensing applications hosted on our LoRa testbed and achieve record-breaking results: (i) highly accurate fine-grained respiration sensing (a subtle $5 \mathrm{~mm}$ chest displacement) at a distance of $15 \mathrm{~m}$ away with a $10 \mathrm{~cm}$-thick brick wall between the target and transceivers; and (ii) accurate tracking of human walking distance and direction even when the target is 30 meters away from the LoRa transceiver pair. Our demo video can be found at: https://youtu.be/-q8XIBdc6GE.

The main contributions of our work are summarized as follows.

- We demonstrate that the new long-range communication technology LoRa can be used for long-range and through-wall contactless wireless sensing.

- We develop the signal propagation model to lay the theoretical foundation for LoRa sensing. Based on this model, we further utilize the ratio of signals received at two antennas to significantly increase LoRa sensing range.

- We conduct comprehensive experiments to evaluate the impact of several important factors on LoRa sensing performance: (a) the effect of target diversity (size, velocity, orientation, etc.), (b) the effect of transceiver pair distance, and (c) the effect of environments (blockage and interference).

- We build a real-time LoRa sensing system with LoRa devices, and demonstrate that our sensing system can effectively monitor both fine-grained human respiration and coarse-grained human walking activities. 


\section{UNDERSTANDING LORA SIGNAL FOR SENSING}

In this section, we first introduce the basics of LoRa, and then develop the LoRa sensing model. At the end, we propose the signal ratio method, which can significantly increase the sensing range of LoRa.

\subsection{LoRa Primer}

LoRa is a physical-layer technology for long-range, low-power wireless communication. LoRa adopts the Chirp Spread Spectrum (CSS) modulation technique [17], which encodes data as frequency varying chirps. The complex exponential representation of a Linear Frequency Modulation (LFM) chirp waveform [24] is given as follows:

$$
\begin{aligned}
S(t) & =\exp \left\{j 2 \pi f_{c} t+j \pi k t^{2}\right\} \\
& =\cos \left(2 \pi f_{c} t+\pi k t^{2}\right)+j \cdot \sin \left(2 \pi f_{c} t+\pi k t^{2}\right),
\end{aligned}
$$

where $f_{c}$ is the central frequency, $k=\frac{B}{T}$ is the sweep rate of the chirp, $B$ is the frequency bandwidth, and $T$ is the chirp duration. The real and imaginary parts of LFM signal can be represented as follows:

$$
\begin{gathered}
I(t)=\cos (\phi(t))=\cos \left(\pi k t^{2}+2 \pi f_{c} t\right), \\
Q(t)=\sin (\phi(t))=\sin \left(\pi k t^{2}+2 \pi f_{c} t\right),
\end{gathered}
$$

where $\phi(t)$ is the instantaneous phase. The instantaneous frequency is $\frac{1}{2 \pi} \frac{d(\phi(t))}{d t}=k t+f_{c}$, which is a linear function of time. Figure 1a depicts the frequency versus time characteristics of a linear FM chirp. The frequency varies linearly from $f_{c}-\frac{B}{2}$ to $f_{c}+\frac{B}{2}$ over $-\frac{T}{2} \leq t \leq \frac{T}{2}$. The corresponding time-domain signal is a cosine based waveform with increasing frequency over time, shown in Figure 1b. Since the chirp fully utilizes its entire allocated bandwidth to encode data, it is more robust against channel noise, multi-path, and doppler effects.

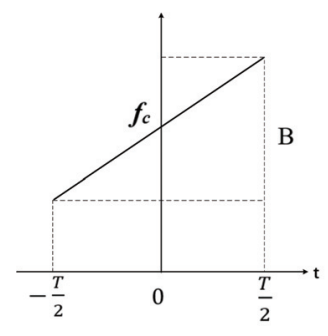

(a) A linear FM chirp

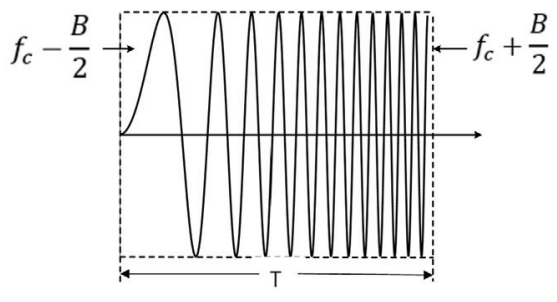

(b) The signal in the time domain

Fig. 1. LFM chirp waveform in the frequency domain (a) and the time domain (b).

\subsection{Modeling LoRa Sensing Process}

We analyze the propagation process of the LoRa signal from a LoRa node (i.e., transmitter) to a LoRa gateway (i.e., receiver). Suppose that a LoRa node generates a chirp signal $s(t)=e^{j \pi k t^{2}}$, then the LoRa digital mixer generates the carrier signal and modulates it with carrier frequency $T x(t)=e^{j \pi k t^{2}+j 2 \pi f_{c} t}$. If a radio signal arrives at the receiver through $N$ different paths (including the direct path and multiple reflected paths from surrounding objects), and the $n^{\text {th }}$ path has a propagation delay of $\tau_{n}(t)$, the received signal can be represented as (the derivation details are presented in Appendix):

$$
R x(t)=e^{j \pi k t^{2}+j 2 \pi f_{c} t} \sum_{n=1}^{N} a_{n}(t) e^{-j 2 \pi f_{c} \tau_{n}(t)},
$$


which describes the signal propagation from multiple paths with corresponding delays, and the superposition of the signals at the receiver. The receiver demodulates the signal with I/Q-demodulation and lowpass filtering. By multiplying the received signal with $e^{-j 2 \pi f_{c}^{\prime} t}$, we obtain the sensing signal as follows:

$$
R x^{\prime}(t)=e^{j\left(\pi k t^{2}+\theta_{c}+\theta_{s}\right)} \sum_{n=1}^{N} a_{n}(t) e^{-j 2 \pi f_{c} \tau_{n}(t)},
$$

where $\theta_{c}=2 \pi \Delta f t$ is the Carrier Frequency Offset (CFO), while $\Delta f=f_{c}-f_{c}^{\prime}$ is caused by unsynchronized clocks at the transmitter and receiver, which has a significant influence on signal phase. $\theta_{s}$ is the phase error introduced by Sampling Frequency Offset (SFO).

Now we employ signal $R x^{\prime}(t)$ for sensing. If there is no moving target in the environment, the received signal is relatively stable as shown in Figure 2c. When an object moves into the sensing area and moves linearly ${ }^{1}$, we observe that the signal envelope shows periodic variations as shown in Figure 2d. Mathematically, the latter part $\sum_{n=1}^{N} a_{n}(t) e^{-j 2 \pi f_{c} \tau_{n}(t)}$ in $R x^{\prime}(t)$ represents the signal envelope which is related to the target's movement.

Note that LoRa signal is not only reflected by the target but also by ambient objects such as walls and furniture. The signal envelope varies with respect to the composition of multiple paths. We further group the multipaths $\left(\sum_{n=1}^{N} a_{n}(t) e^{-j 2 \pi f_{c} \tau_{n}(t)}\right)$ into static multipaths and dynamic multipath as shown in Figure 2a. All the multipaths from static objects and walls can be represented as a combined single static vector $H_{s}=\sum_{i \in P_{s}} a_{i} e^{j 2 \pi f \tau_{i}(t)}$, i.e., static vector in the $\mathrm{I} / \mathrm{Q}$ space. For the dynamic multipath, the moving target will cause the path length of the reflected signal to vary and we call it dynamic vector $H_{d}=a(t) e^{-j 2 \pi f_{c} \tau(t)}, \tau(t)=\frac{d(t)}{c}$, where $d(t)$ is the length of the dynamic path, $c$ is the speed of light ${ }^{2}$. The dynamic component rotates with the static component (Figure $2 b$ ), inducing the signal envelope variations shown in Figure $2 \mathrm{~d}$. Therefore, the received signal can be represented as

\footnotetext{
${ }^{1}$ The target moves along a sliding track with a length of $1 \mathrm{~m}$. The spacing between transceivers is $1 \mathrm{~m}$. ${ }^{2} f_{c}=915 \mathrm{MHz}, c=3 \times 10^{8} \mathrm{~m} / \mathrm{s}, \lambda=c / f_{c}=0.3279 \mathrm{~m}$.
}

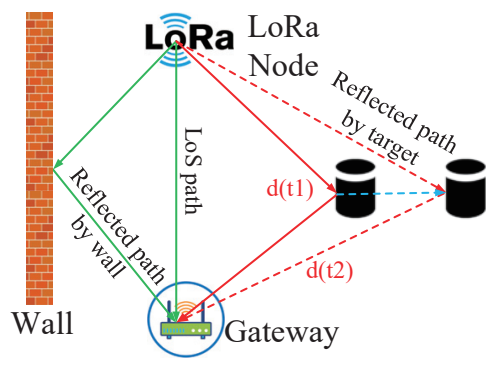

(a) Scenario

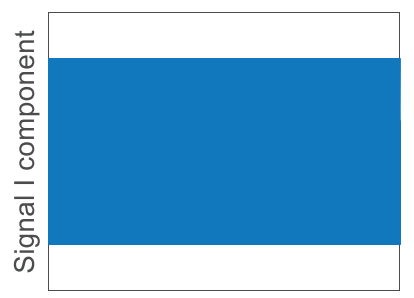

(c) Signal without moving target

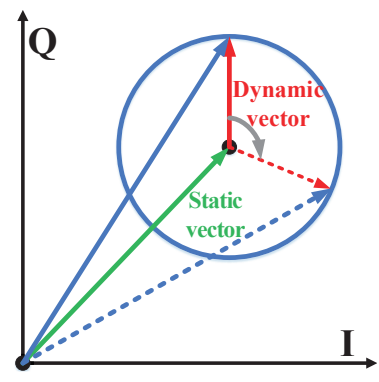

(b) I/Q Vector Representation

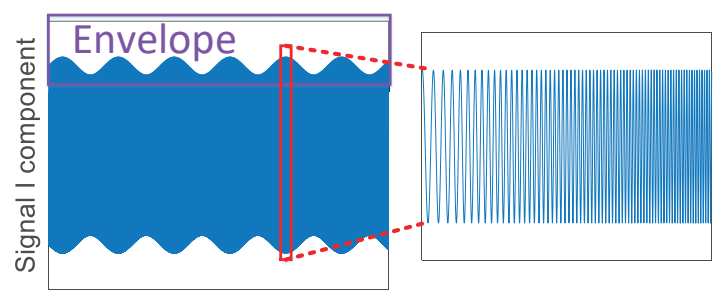

(d) Signal with moving target

Fig. 2. The received signal with/without moving target in the environment. 
follows:

$$
R x^{\prime}(t)=e^{j\left(\pi k t^{2}+\theta_{c}+\theta_{s}\right)}\left(H_{s}+a(t) e^{-j \frac{2 \pi d(t)}{\lambda}}\right)
$$

\subsection{Employing the Ratio of Received Signals}

As mentioned earlier, the chirp with entire allocated bandwidth makes the signal robust against interferences during the propagation process. After receiving the signal, we need to remove the high frequency component and extract the signal envelope, which contains the movement information of the target. The challenge is, due to $\mathrm{CFO}$ and other offset errors, the phase measurement is random and thus can not be applied for sensing.

We note that the LoRa gateway is typically equipped with two antennas [20]. The two antennas share the same clock, and thus have the same CFO and SFO phase offsets. This offers us an opportunity to remove the varying random frequency offsets. Based on this key observation, we propose to utilize the ratio of the two signals received simultaneously at the two antennas, which can be derived as follows:

$$
S R(t)=\frac{R_{1}(t)}{R_{2}(t)}=\frac{H_{s 1}+a_{1}(t) e^{-j \frac{2 \pi d(t)}{\lambda}}}{H_{s 2}+a_{2}(t) e^{-j \frac{2 \pi(d(t)+\Delta s)}{\lambda}}},
$$

where $R_{1}(t)$ and $R_{2}(t)$ are the signals received at two different antennas. The varying frequency offsets are removed by this division operation. $H_{s 1}(t)$ and $H_{s 2}(t)$ are the static signal components, while $a_{1}(t)$ and $a_{2}(t)$ are the attenuation and initial phase offsets of the two antennas' dynamic path components, respectively. Compared with $d(t), \Delta s$ caused by different physical locations of the two antennas is a much smaller distance, which can be considered as a constant within a short period of time.

We focus on the dynamic path length $d(t)$, which is related to the target's movement. The target-induced phase shift due to dynamic path is represented as $\eta$ :

$$
\eta=e^{-j \frac{2 \pi d(t)}{\lambda}}
$$

The constant parameters include $a=a_{1}(t), b=H_{s 1}, c=a_{2}(t) e^{-j \frac{2 \pi \Delta s}{\lambda}}$ and $d=H_{s 2}$. The ratio of the two received signals can now be simplified as:

$$
S R(t)=\frac{R_{1}}{R_{2}}=\frac{a \eta+b}{c \eta+d}=\frac{b c-a d}{c} \frac{1}{c \eta+d}+\frac{a}{c}
$$

In essence, the signal ratio can be decomposed into a series of fractional linear transformations of the signal at one antenna, also referred to as Möbius transformation [60], which includes rotation operation (i.e., $c \eta$ ), translation operation (i.e., $c \eta+d$ ), and inversion operation (i.e., $\frac{1}{c \eta+d}$ ). As shown in Figure 3a, these transformations do not change the shape and orientation but only the position and size of the rotation trajectory (the dynamic signal vector rotates with respect to the static signal vector). Thus, the original static/dynamic vector model can still be applied when we employ the signal ratio for sensing. The difference is that we now have a scaled new dynamic component rotating with respect to the scaled new static component as shown in Figure 3a. The signal ratio can be presented by the following equation:

$$
S R(t)=F(\eta)=F\left(e^{-j \frac{2 \pi d(t)}{\lambda}}\right)
$$

This division operation removes the varying frequency offsets and the phase is now stable. Figure $3 \mathrm{~b}$ shows the amplitude of the received signal ratio when the target is moving. The signal shows clear periodical variations, and we also observe that the high frequency component is cancelled out. 


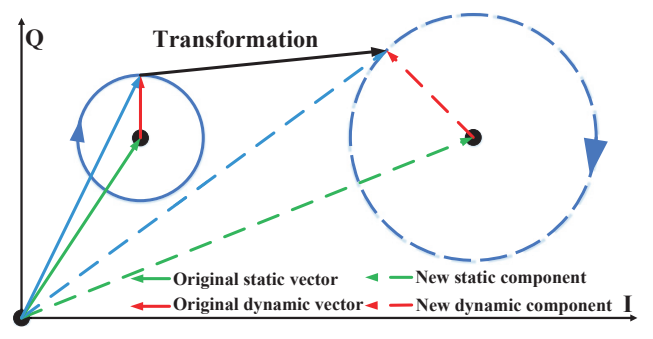

(a) I/Q Vector Representation

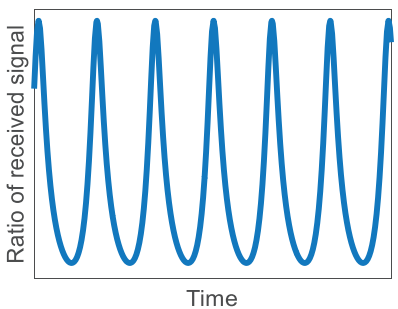

(b) Amplitude of signal ratio

Fig. 3. Utilizing signal ratio for LoRa sensing.

\subsection{Quantify the Phase Change of Signal Ratio}

Next, we describe in detail how to employ signal ratio to sense the moving target. Based on the representation of the received signal ratio $S R(t)=F(\eta)=F\left(e^{-j \frac{2 \pi d(t)}{\lambda}}\right)$, the phase change of the signal ratio induced by a moving target is the same as that of the original signal from one antenna, which can be written as:

$$
\Delta \phi=-2 \pi \frac{d(t 2)-d(t 1)}{\lambda} \approx-2 \pi \frac{2 \Delta d}{\lambda}
$$

Here $d(t 1)$ and $d(t 2)$ are the reflection path lengths of the start and end positions of the moving target. $\Delta d$ is the moving distance of the target, the reflection path length change is $2 \Delta d$ when the target is far away from the transceivers. If the target moves continuously by half a wavelength $\frac{\lambda}{2}$, the resultant reflected signal will exhibit a phase change (rotation) of $2 \pi$. By measuring the phase change $\Delta \phi$ of the dynamic component, we can obtain the target movement distance $\Delta d$ as follows:

$$
\Delta d \approx-\frac{\Delta \phi}{4 \pi} \lambda
$$

The phase change $\Delta \phi$ of the dynamic component is thus the key for target movement distance estimation. Note that at the receiver side, the dynamic component and static component are mixed together. We need to remove the static component before we can estimate the phase change of the dynamic component. One method adopted in $\mathrm{WiFi}$ sensing is to first estimate the static component by averaging the data samples over a relatively long sliding window, and then remove it from the mixed signal to obtain the dynamic component. In LoRa sensing, when the target is far away from the sensing device, the static component can still change slightly over time. If we employ a relatively large window size, the static component can not be assumed to be a constant any more. If we employ a small window size, without knowing the start and end sample points, the static component can not be estimated accurately. In this paper, we propose a new method to accurately estimate the phase change of the dynamic component without the need of calculating the static components. We define the vector on the tangential direction of the dynamic component as $H_{t}$, which is calculated by subtracting a sample point from its immediate successor. The signal ratio samples we obtained are the combined one with both static and dynamic components which are represented as $H_{c}$. As shown in Figure $4 \mathrm{a}, H_{c}$ and $H_{c}^{\prime}$ are two adjacent signal ratio samples in the complex plane. $H_{d}, H_{d}^{\prime}$ and $H_{s}$ are the dynamic and static components of these two nearby data points, respectively. We can now derive the mathematical expression for the vector $H_{t}$ on the tangential direction of the dynamic component $H_{d}$ by calculating the difference between two adjacent samples as follows:

$$
H_{t}=H_{c}^{\prime}-H_{c}=\left(H_{s}+H_{d}^{\prime}\right)-\left(H_{s}+H_{d}\right)=H_{d}^{\prime}-H_{d}
$$




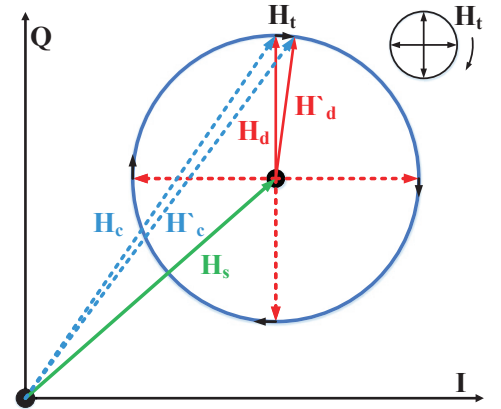

(a) Obtaining vector on the tangential direction

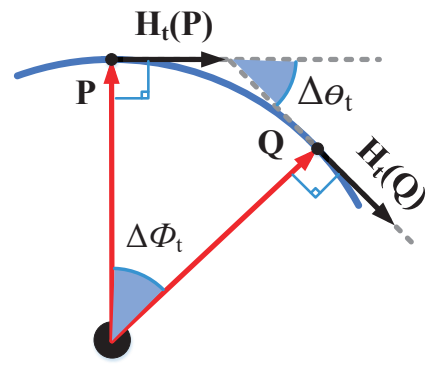

(b) Phase change is measured by slope of vector on the tangential direction

Fig. 4. Deriving the phase change of signal ratio.

The sample difference ${ }^{3}$ can help cancel out the common static multipath component. This is because adjacent samples are very close ${ }^{4}$ to each other in the time domain and thus the static component in both samples can be safely considered as the same. The remaining part is the difference of the two dynamic components, which is a vector on the the tangential direction of the dynamic component. Now we obtain a new metric of tangential vector to measure the phase change of the dynamic component. As shown in Figure $4 \mathrm{~b}$, the dynamic component rotates with a phase change of $\Delta \phi_{t}$ from point $P$ to $Q$. For the two points $P$ and $Q$ on the circle, the vectors on the tangential direction are defined as $H_{t}(P)$ and $H_{t}(Q)$, respectively. The phase change between vectors $H_{t}(P)$ and $H_{t}(Q)$ is $\Delta \theta_{t}$. We observe that the phase change of the tangential vectors $\Delta \theta_{t}$ equals exactly to the dynamic component shift $\Delta \phi_{t}$. By calculating the phase difference of vector $H_{t}(P)$ and $H_{t}(Q)$, we obtain the phase change of the dynamic component: $\Delta \phi_{t}=\Delta \theta_{t}=\phi_{P}-\phi_{Q}$. By cumulating the phase change of the tangential vectors, we are able to measure the phase changes of the dynamic components and accordingly estimate the distance of the target movement. We employ an example to illustrate how the distance is calculated. We first obtain the complex representation of the signal ratio processed with the Savitzky Golay filter [46], and then calculate the difference between two adjacent samples to obtain the tangential vectors. We can see that the vector rotates in the complex plane as shown in Figure 5a. We then calculate the phase difference of two tangential vectors separated by a certain interval ${ }^{5}$ (e.g., $0.01 \mathrm{~s}$ ), which reflects the corresponding phase change of the dynamic components in Figure $5 \mathrm{~b}$. We filter out the small phase variation with linear interpolation. We observe the total accumulated phase change of the dynamic component is $38.5=6.1 \times 2 \pi$ in Figure $5 \mathrm{c}$, which indicates a moving distance of $d=\frac{6.1 \times 2 \pi}{4 \pi} \times 0.33=1.01 \mathrm{~m}\left(\lambda=\frac{C}{f}=\frac{3 \times 10^{8} \mathrm{~m} / \mathrm{s}}{915 \mathrm{MHz}}=0.33 \mathrm{~m}\right)$. This result matches the $1 \mathrm{~m}$ ground-truth very well. This example demonstrates that we can employ the fine-grained phase change of signal ratio to estimate target movement accurately.

\section{EXPLORING LORA SENSING CAPABILITY}

In this section, based on the sensing model and techniques proposed, we design a series of experiments to comprehensively explore the sensing capability of LoRa. We conduct experiments to evaluate the impact of

\footnotetext{
${ }^{3}$ We employ adjacent LoRa data samples with one millisecond time span in between.

${ }^{4}$ The sampling rate can be up to hundreds of $\mathrm{kHz}$.

${ }^{5}$ Note that this time interval needs to be chosen to make sure the amount of phase change of the dynamic vector is smaller than $2 \pi$. For a $1 \mathrm{~m} / \mathrm{s}$ moving speed, the target moves only $0.01 \mathrm{~m}$ in $0.01 \mathrm{~s}$ and the phase change of the dynamic vector is well below $0.1 \pi$.
} 


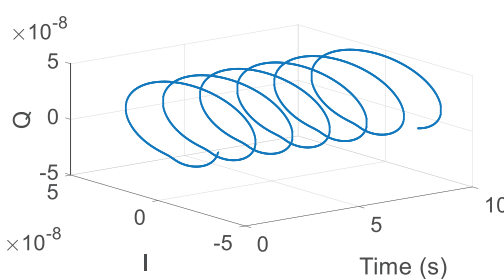

(a) Vectors on the tangential direction in(b) Phase change of dynamic compocomplex plane

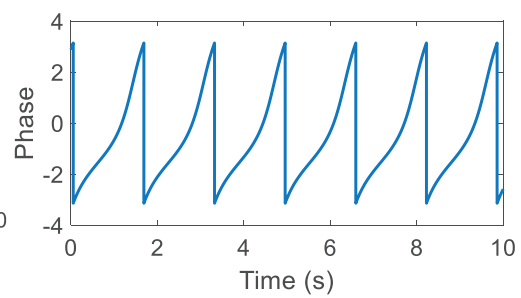

nents

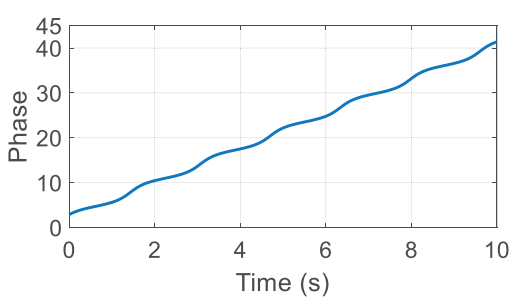

(c) Unwrapped phase change

Fig. 5. The phase change is measured by vector on the tangential direction.

several important factors on the sensing performance: (a) the effect of target diversity (size, velocity, orientation, etc.), (b) the effect of distance between the LoRa transceivers and (c) the effect of environments (interference and blockage). The organization of this section is as follows: In Section 3.1, we clearly describe our prototype implementation, experimental setup and target. We first conduct a preliminary study on sensing range and sensing granularity in Section 3.2. Then, we investigate the effect of target diversity including target moving distance and orientation, target size, target moving speed, etc., in Section 3.3. We vary the spacing between the transceiver pair to see the effect on sensing performance in Section 3.4. At last, we evaluate the effect of environments, including blockage (i.e., through-wall sensing), and interference in Section 3.5.

\subsection{Prototype Implementation and Experimental Setup}

As shown in Figure 6a and 6b, our LoRa sensing prototype is composed of one LoRa transmitter and one LoRa receiver. The LoRa transmitter is Semtech SX1276 [7] with an Arduino Uno [3], equipped with one antenna. We configure the LoRa node to transmit signal at the $915 \mathrm{MHz}$ frequency band with a $125 \mathrm{kHz}$ channel bandwidth. Our LoRa gateway is based on USRP B210 [2] and GNU Radio [1]. The gateway is equipped with two antennas. In our experiments, we test both directional and omnidirectional antennas. We choose the commonly-used directional antennas $(25 \times 22 \mathrm{~cm})$ with a $70^{\circ}$ azimuth beamwidth and a $8 \mathrm{dBi}$ gain. For the omnidirectional antenna, the gain is $3 \mathrm{dBi}$. We set the sampling rate of the USRP to $800 \mathrm{kHz}$. A Lenovo R720 laptop with an Intel Core i7 CPU and 16GB RAM is connected to the receiver via a USB cable to collect packet samples and process the data.

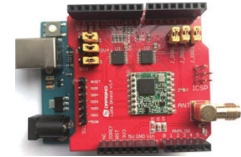

(a) LoRa node

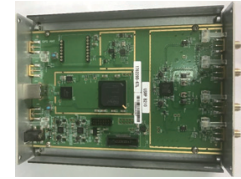

(b) USRP-based gateway
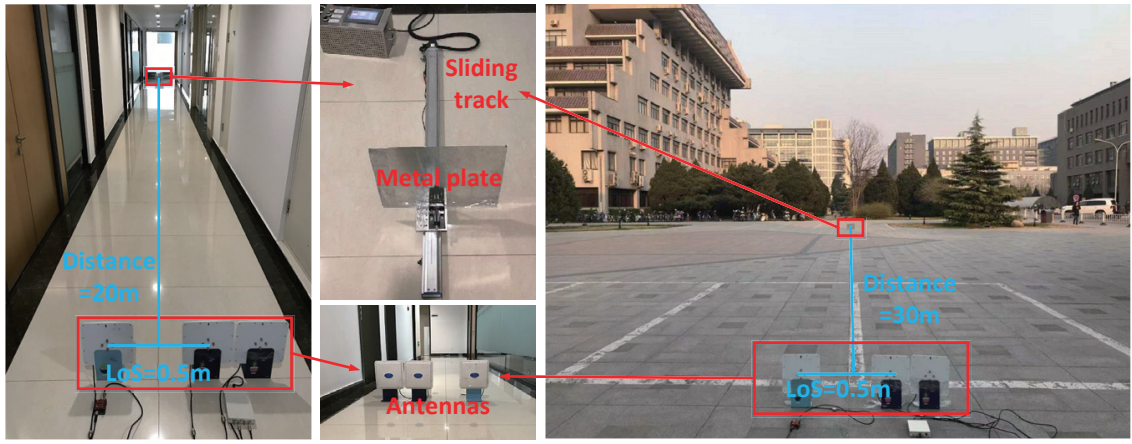

(c) Test environments

Fig. 6. Experimental setup.

We conduct experiments with both directional antenna and omnidirectional antenna for LoRa sensing and we find directional antenna is preferred for sensing due to the following two reasons: (i) it reduces the amount 
of interference from surroundings and (ii) it increases the sensing range. We present the comparison results in Section 3.5.2 and directional antennas are used in our experiments unless otherwise specified.

We conduct experiments in both indoor and outdoor environments, as shown in Figure 6c. We use a metal plate (the ideal signal reflector) as the target to verify the sensing performance. The metal plate has a size of $35 \mathrm{~cm} \times 40 \mathrm{~cm}$ and we employ a $1 \mathrm{~m}$-long sliding track controlled by Raspberry Pi 3 Model to precisely move the metal plate.

\subsection{Preliminary Study on LoRa Sensing}

3.2.1 LoRa Sensing Range. We first validate the long distance sensing capability of LoRa in both indoor and outdoor environments and compare the results with WiFi sensing. For WiFi sensing, we employ Gigabyte GB-BXi5-4570R mini-PCs equipped with Intel $5300 \mathrm{WiFi}$ cards and omnidirectional antennas as transceivers.

Experiment 1: We consider both small-scale motion ( $5 \mathrm{~mm}$ displacement) and large-scale motion (1m displacement) in our experiments. The metal plate moves on the sliding track with precisely controlled amount of displacements. In the indoor environment, we place the metal plate at a distance of $10 \mathrm{~m}$ to $50 \mathrm{~m}$ at a step size of $5 \mathrm{~m}$ from the transceivers. The spacing between the transceivers is set to $0.5 \mathrm{~m}$. We perform the test at each distance until we are not able to clearly detect the movement. The sensing range limits are shown in Table 2 . We can see that LoRa signal is capable of sensing even a subtle $5 \mathrm{~mm}$ displacement at a distance of $25 \mathrm{~m}$ away in the indoor environment. On the other hand, the sensing limit of WiFi signal is only around $5 \mathrm{~m}$. For large scale $(1 \mathrm{~m})$ motions, the sensing distance of LoRa can reach up to $50 \mathrm{~m} .{ }^{6}$ In the outdoor environment, we observe slightly shorter sensing ranges which are $18 \mathrm{~m}$ ( $5 \mathrm{~mm}$ displacement) and $35 \mathrm{~m}$ ( $1 \mathrm{~m}$ displacement), respectively. These results are different from our expectations. One possible reason could be that in the indoor environment, we have stronger reflections from the walls and ceilings and these static reflections actually strengthen the overall received signal at the receiver. Thus the sensing distance is larger in the indoor environment. We further evaluate the sensing range limit when omnidirectional antennas are used in LoRa. We find that the sensing distance is decreased from $50 \mathrm{~m}$ to $15 \mathrm{~m}$ for large-scale movement in the indoor environment. We can see that even when LoRa and WiFi both employ omnidirectional antennas, the sensing range of LoRa is still much larger than that of WiFi.

Table 2. Sensing Range Verification and Comparison in Indoor/Outdoor Environments with Small/Large-scale Movement

\begin{tabular}{|c|c|c|c|c|}
\hline & \multicolumn{2}{|c|}{ Indoor Environment } & \multicolumn{2}{c|}{ Outdoor Environment } \\
\hline Movement & Small Scale & Large Scale & Small Scale & Large Scale \\
\hline WiFi & $5 \mathrm{~m}$ & $8 \mathrm{~m}$ & $5 \mathrm{~m}$ & $9 \mathrm{~m}$ \\
\hline LoRa & $25 \mathrm{~m}$ & $50 \mathrm{~m}$ & $18 \mathrm{~m}$ & $35 \mathrm{~m}$ \\
\hline
\end{tabular}

3.2.2 LoRa Sensing Granularity. We study the sensing granularity using a metal plate when it is placed far away. We notice that for subtle movements, the movement-induced signal variation is small. The situation is even worse when the target is far away and the small variation can be easily buried in noise. We find that for small-scale displacement, our proposed signal ratio method is particularly efficient. Figure 7 shows the signal variations ${ }^{7}$ when the target is $25 \mathrm{~m}$ away and the displacement is $5 \mathrm{~mm}$. Figure 7 a shows the signal variation with the signal received at one antenna (adopted in existing works) and Figure 7b shows the variation of the signal ratio (our proposed method). We can clearly see that the amplitude of signal ratio exhibits much clearer periodic variations compared with the received signal. This is because signal ratio cancels out noise and the value is also normalized to a larger value, which increases the sensing range and is particularly efficient for sensing subtle movements.

\footnotetext{
${ }^{6}$ Due to space restriction in the indoor environment, we stop at $50 \mathrm{~m}$.

${ }^{7}$ The signals are processed with the Savitzky Golay filter [46].
} 


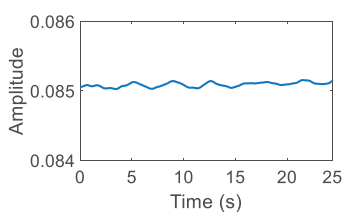

(a) Received signal

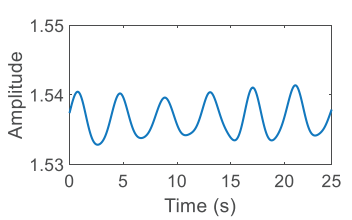

(b) Signal ratio

Fig. 7. Comparison of induced signal variation.

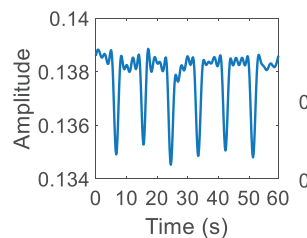

(a) $5 \mathrm{~mm}$

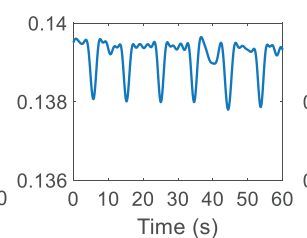

(b) $2 \mathrm{~mm}$

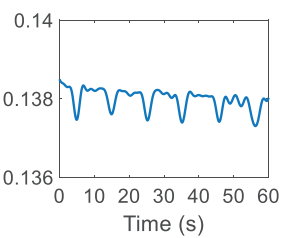

(c) $1 \mathrm{~mm}$

Fig. 8. Signal ratio for sensing subtle vibrations of target.

Experiment 2: To obtain the sensing granularity limit of LoRa, we place the metal plate at $25 \mathrm{~m}$ away from the transceivers. Then we move the metal plate back and forth with $5 \mathrm{~mm}, 2 \mathrm{~mm}$ and $1 \mathrm{~mm}$ displacements on the sliding track, respectively. Figure 8 shows the amplitude variations of signal ratio for these three displacements. We can clearly identify that the target moves forward and backward for six times. When we reduce the displacement further to $0.5 \mathrm{~mm}$, we sometimes are not able to see clear variation patterns on signal ratio. So we conclude that at a distance of $25 \mathrm{~m}, \mathrm{LoRa}$ is capable of sensing a small $1 \mathrm{~mm}$ displacement, which is even smaller than the respiration-caused chest displacement (around $5 \mathrm{~mm}$ ). We evaluate the performance of long distance respiration sensing with real human targets in Section 4.

\subsection{Effect of Target Diversity}

In this subsection, we investigate the effect of target diversity, including target moving distance and orientation, target size, and target moving speed.

3.3.1 Effect of Target Moving Distance and Target Location. Based on the modelling in Section 2, we know that the dynamic component of signal ratio rotates with respect to the static component, and the amount of rotation is determined by the distance the target moves. For example, if we observe 6.1 cycles of cosine waveform in signal ratio, the moving distance of the target can be calculated as $d=\frac{6.1 \times 2 \pi}{4 \pi} \times 0.33=1 \mathrm{~m}$. Similarly, if the target moves less than $\frac{\lambda}{2}$, the phase rotation is also less than a full cycle. As a result, the observed amplitude change of signal ratio is just a fragment of a complete sinusoidal cycle. As shown in Figure 9, depending on where the phase rotation starts on the sinusoidal cycle, the same amount of target movement can cause different patterns of amplitude variations. We further discover where the phase rotation starts depends on the relative location of the target with respect to the transceiver pair. Thus, the target location does affect the sensing performance and a small location change can change the amount of signal variations and accordingly the sensing performance.

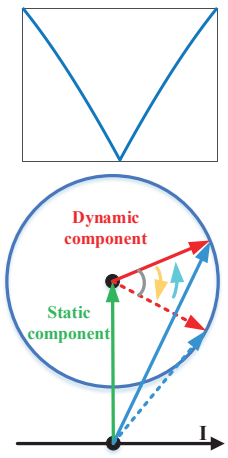

(a)

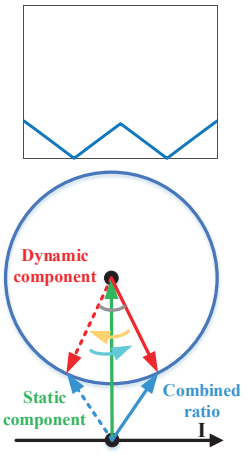

(b)

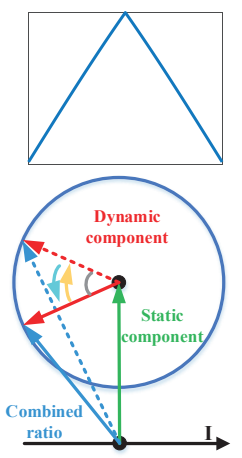

(c)

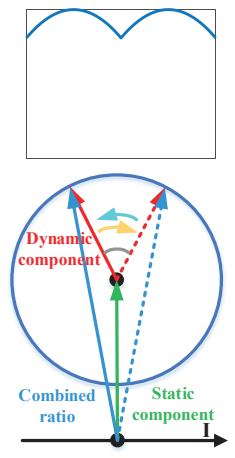

(d)

Fig. 9. Signal pattern when target moves less than half a wavelength with different starting location. 
Experiment 3: We study the effect of target moving distance on the received signal ratio. Without loss of generality, in an indoor environment, we place the metal plate at $20 \mathrm{~m}$ away from the transceivers. The LoRa transceivers are separated by $0.5 \mathrm{~m}$. We employ the sliding track to move the metal plate for $1 \mathrm{~m}$ (large-scale motion) and $5 \mathrm{~mm}$ (small-scale motion), respectively. Figure 10a shows the signal ratio in complex plane throughout the process. We observe that a $1 \mathrm{~m}$ movement induces a phase rotation of $12.2 \pi\left(\frac{2 \times 1}{0.3279} \times 2 \pi\right)$. Thus the amplitude of signal ratio shows 6.1 cycles of change, which matches the theoretical result in Figure 3b. On the other hand, when the metal plate moves back and forth with a $5 \mathrm{~mm}$ displacement, the observed signal change is only a fragment of the complete sinusoidal cycle, as shown in Figure 10c.

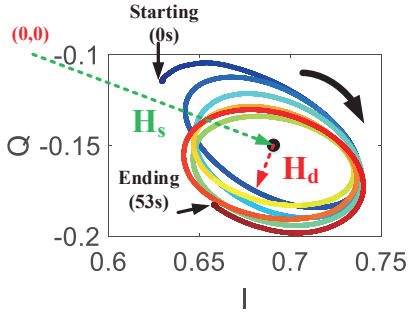

(a) $1 \mathrm{~m}: \mathrm{I} / \mathrm{Q}$ trace of signal ratio

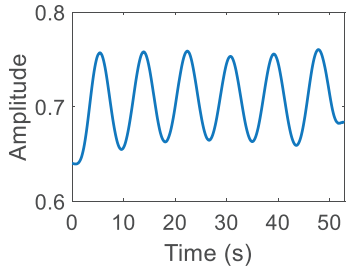

(b) $1 \mathrm{~m}$ : amplitude of signal ratio

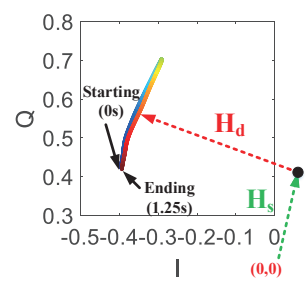

(c) $5 \mathrm{~mm}: \mathrm{I} / \mathrm{Q}$ trace of signal ratio

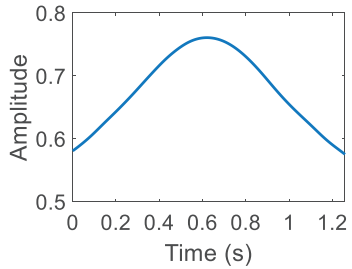

(d) $5 \mathrm{~mm}$ : amplitude of signal ratio

Fig. 10. Utilizing signal ratio for LoRa sensing: I/Q trace and amplitude of signal ratio for $1 \mathrm{~m}$ and $5 \mathrm{~mm}$ displacements.

3.3.2 Effect of Target Moving Direction. Next we investigate the effect of target moving orientation on sensing. We consider the two extreme cases when the target moves in parallel $\left(0^{\circ}\right)$ and perpendicular $\left(90^{\circ}\right)$ with respect to the two transceivers. Other directions are between these two extreme cases.

Experiment 4: The metal plate moves $1 \mathrm{~m}$ on the sliding track forward and backward with respect to the transceiver pair. When the target moves perpendicular $\left(90^{\circ}\right)$ to the transceiver pair, we notice that if the target moves towards the transceivers, the dynamic component rotates with respect to the static component counterclockwise. When the target moves away from the transceivers, the dynamic component rotates clockwise, as shown in Figure 11. During the movement process (from Location 1 to Location 2 and then back), we observe that the dynamic component first rotates counterclockwise, and then rotates clockwise back to its starting point as shown in Figure 12.
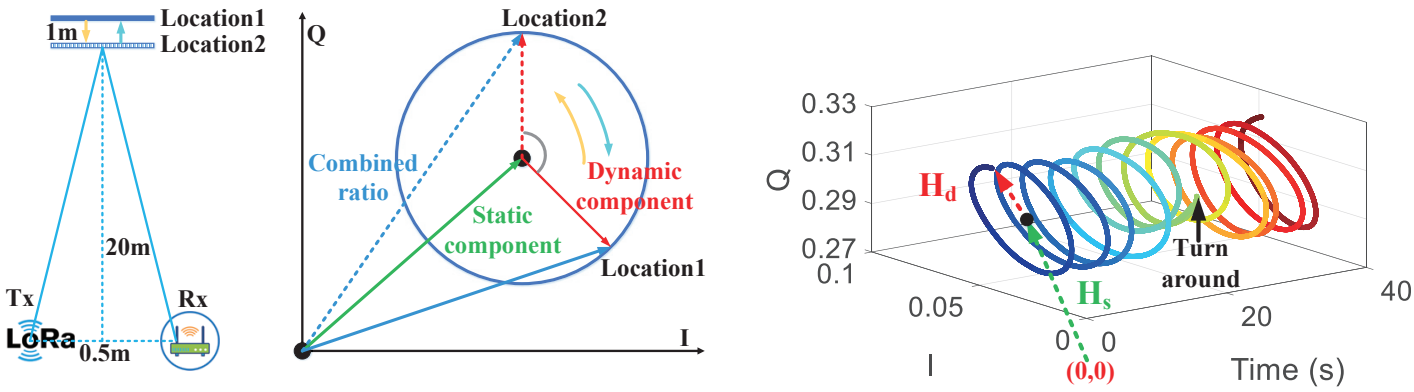

Fig. 11. Target moving direction with corresponding dynamic component rotation direction.

Fig. 12. $\mathrm{I} / \mathrm{Q}$ trace in the complex plane. 
Next we investigate how the signal changes when the target moves in parallel to the transceiver pair. The spacing between the transceivers is set to $0.5 \mathrm{~m}$, and the metal plate is $10 \mathrm{~m}$ away. When the target moves across the perpendicular bisector of the transceiver pair, the reflection path first becomes shorter and then longer. The path length changes as follows: $[20.0312 \mathrm{~m}-20.0062 \mathrm{~m}-20.0312 \mathrm{~m}]$. The reflection path difference is only $2.5 \mathrm{~cm}$ with a $1 \mathrm{~m}$ movement. Based on these results, we know that, with different orientations, the same amount of travel distance induces very different reflection path length change, and thus different signal variation patterns.

3.3.3 Effect of Target Size and Moving Speed. We further investigate the impact of target size and moving speed on the sensing performance.

Experiment 5: We place the metal plate at $10 \mathrm{~m}$ away. The spacing between transceivers is set to $1 \mathrm{~m}$. We employ two metal plates of different sizes $(35 \times 40 \mathrm{~cm}$ and $10 \times 15 \mathrm{~cm})$ to see the difference. We observe that clear signal variations can be obtained with both metal plates. The large metal plate achieves larger signal variations than the small one. So a larger surface does cause stronger reflection and accordingly better sensing performance. We then vary the target moving speed from fast $(0.077 \mathrm{~m} / \mathrm{s})$ to medium $(0.030 \mathrm{~m} / \mathrm{s})$ and finally slow $(0.015 \mathrm{~m} / \mathrm{s})$ to see the effect on sensing. Figure 13 shows the signal variations when the target moves at three different speeds. We can see that the speed difference only causes the signal variation frequency to change but not the variation amplitude. For sensing capability, we care about the variation amplitude and thus the speed does not affect the sensing performance much. However, if the speed is very high (meters per second), we expect the Doppler shift will then be big and may affect the phase readings and accordingly affect the sensing performance.

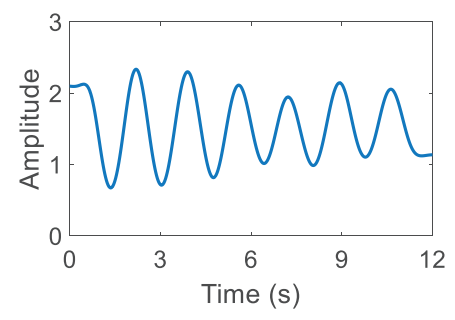

(a) Large plate

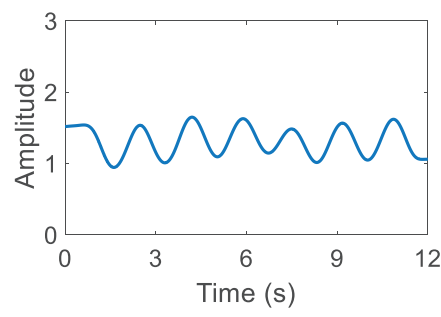

(b) Small plate

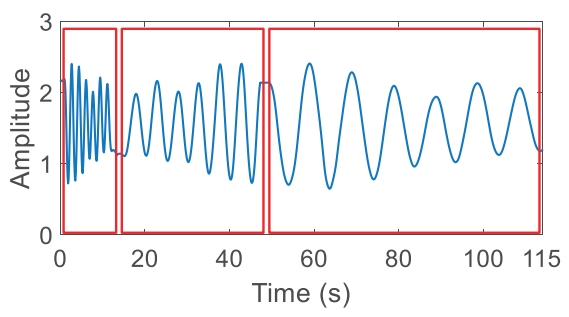

(c) Signal ratio with different moving speeds

Fig. 13. Effect of target size and moving speed on sensing performance.

\subsection{Effect of Spacing Change between Transceiver Pair}

In this subsection, we investigate the impact of spacing between the transceivers on sensing. When we change the spacing between the transceivers, the length of the reflection path changes.

Experiment 6: As shown in Figure 14, we vary the spacing between the transmitter and receiver as $1 \mathrm{~m}$, $2 \mathrm{~m}$, and $3 \mathrm{~m}$. The target is $10 \mathrm{~m}$ away and moves $1 \mathrm{~m}$ on the sliding track. The path length changes are $1.9977 \mathrm{~m}$, $1.9909 \mathrm{~m}$, and $1.9799 \mathrm{~m}$ for the three scenarios. Note that the same $1 \mathrm{~m}$ movement induces different amounts of path length changes and accordingly different amounts of phase rotations. We obtain $\phi_{1}=2 \pi \frac{1.9977}{0.3279}=12.1848 \pi$, $\phi_{2}=2 \pi \frac{1.9909}{0.3279}=12.1433 \pi$, and $\phi_{3}=2 \pi \frac{1.9799}{0.3279}=12.0762 \pi$ phase changes for the three spacings, respectively. When the spacing is $1 \mathrm{~m}$, the phase rotation is $0.1086 \pi$ more than when the spacing is $3 \mathrm{~m}$. The results match our theoretical model. The varying transceiver spacing causes slightly different phase changes. Also the varying spacing causes slightly different rotation starting positions. Hence the signal ratio variations are slightly different as shown in Figure 14d-14f but all have 6 cycles of change. 


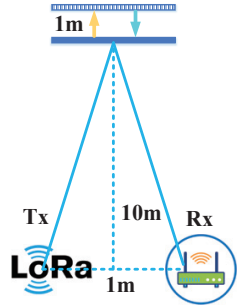

(a) $\operatorname{LoS}=1 \mathrm{~m}$

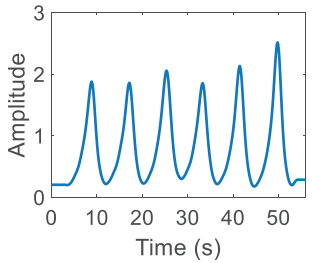

(d) $\operatorname{LoS}=1 \mathrm{~m}$

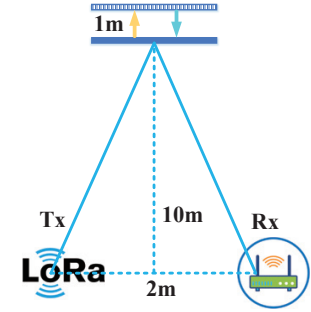

(b) $\operatorname{LoS}=2 \mathrm{~m}$

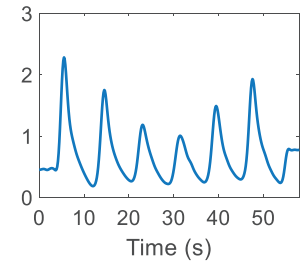

(e) $\operatorname{LoS}=2 m$

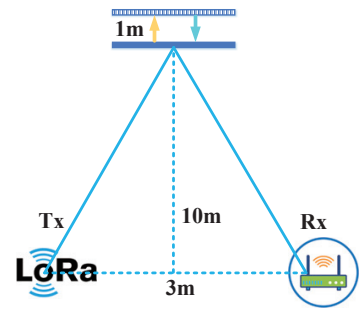

(c) $\operatorname{LoS}=3 \mathrm{~m}$

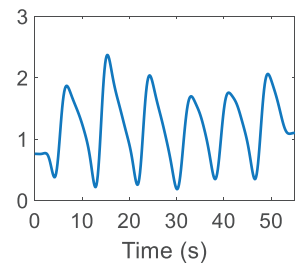

(f) $\operatorname{LoS}=3 m$

Fig. 14. The received signals under different LoS.

\subsection{Effect of Environments}

In this subsection, we investigate the effect of environments, including blockage (i.e., through-wall sensing) and interference.

3.5.1 Through-wall Sensing. We know that LoRa signal has a strong capability of penetrating through walls. This provides unique opportunities for through-wall sensing in application scenarios such as disaster rescue.

Experiment 7: We place the metal plate and the LoRa transceivers in different rooms. The distance between the target and the transceivers is set to $10 \mathrm{~m}$ with one wall in between. The wall is made of bricks and has a thickness of $10 \mathrm{~cm}$. The metal plate moves $1 \mathrm{~m}$ on the sliding track. In this scenario, we observe clear signal variation caused by the target movement in Figure 15b. Then we increase the distance to $15 \mathrm{~m}$ with two walls between the target and transceiver pair. We can still observe the expected variation pattern with periodic cycles. We further test the small scale $5 \mathrm{~mm}$ movement in both scenarios. We find that we can clearly detect the $5 \mathrm{~mm}$ displacement in Scenario 1 (10m with one wall) and also in Scenario 2 (15m with two walls).

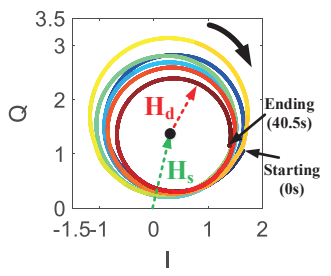

(a) Signal ratio in the complex plane (one wall)

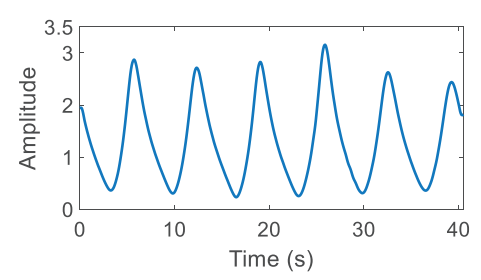

(b) Amplitude of signal ratio (one wall)

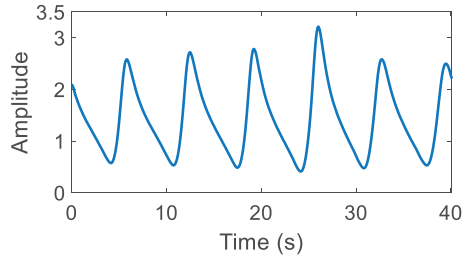

(c) Amplitude of signal ratio (two walls)

Fig. 15. Effect of sensing through one wall and two walls.

3.5.2 Interferences from Surroundings. Now we study the interferences from other moving objects in the environment. We consider both directional antenna and omnidirectional antenna for sensing. 
Experiment 8: We let the metal plate move $1 \mathrm{~m}$ on the sliding track and place the target $10 \mathrm{~m}$ away from the transceivers. Then one person acting as the interferer randomly walks around at different positions. We observe that if we employ the omnidirectional antenna, the sensing performance will be severely interfered even when the interferer is not close to the target, and there is interference in almost all 360 degree directions. In contrast, when we switch to directional antennas with a 70 degree beamwidth, we observe that when the interferer is 40 degrees off the central beam direction, the interference is small enough to be neglected. However, if the interferer is in the same direction of the beam, even when the interferer is 20 meters away from the target, it still interferes with the target. From this experiment, we can see that directional antenna is a preferred option to mitigate the interference for LoRa sensing. However, even with directional antenna, the problem is not fully solved. One way to address this issue is to convert the signal into the frequency domain. A lot of movements have specific frequency ranges. For example, the frequency of human respiration is in the range of 0.10 to $0.33 \mathrm{~Hz}$ while a machine's vibration has a much higher frequency and thus can be easily separated in the frequency domain. Another way of addressing the interference is to separate the signal of interest from those interfering signals in time domain with multi-dimensional information (i.e., angle-of-arrival, time-of-arrival and Doppler shift) [57].

In summary, the objective of these experiments is to show the sensing performance of LoRa under various conditions and also explore the limitations and boundaries of LoRa sensing. These results lay a solid foundation for our real-life human target sensing application in next section. We notice that it is still very difficult for LoRa to deal with multi-target sensing in time domain as it is very challenging to separate multiple signals in time domain with a limited bandwidth. However, it is possible to employ multiple antennas to deal with multi-target sensing in spatial domain. As Semtech has already planned to develop LoRa gateways with multiple antennas (4-8), we believe it is feasible to employ the antenna array available in the future to enable multi-target sensing.

\section{APPLICATIONS AND EVALUATION}

In this section, we build a LoRa sensing prototype to realize two applications: (1) long-range through-wall respiration sensing and (2) long-range through-wall human tracking (both distance and orientation). Our system consists of a pair of LoRa node and gateway. The LoRa node (Arduino Uno [3] is equipped with Semtech SX1276 [7]) is configured to transmit signals at $915 \mathrm{MHz}$ frequency band with a $125 \mathrm{kHz}$ channel bandwidth. The LoRa gateway is built based on USRP B210 [2] and GNU Radio [1], which is equipped with two commonly-used directional antennas. The LoRa gateway is connected to the data processing laptop with an Intel Core i7 CPU and 16GB RAM, which collects 1000 data samples per second ${ }^{8}$ and processes them with MATLAB in real time. The demo video can be found at: https://youtu.be/-q8XIBdc6GE.

\subsection{Long-range Through-wall Respiration Sensing}

In this subsection, we first present the implementation of a real-time respiration monitoring system. Then we evaluate the effectiveness of respiration sensing in challenging real-life scenarios where the transceivers are located far away from the target and behind walls. We also evaluate the system performance with changing LoS distances, different human targets, and different indoor environments. Finally, we discuss the interference from the surrounding environment when sensing respiration at a long distance.

4.1.1 System Implementation. To monitor human respiration, we first obtain two signal streams (one from each antenna) simultaneously to calculate the signal ratio. We process the signal data with a 30s sliding window in real time. A band pass filter is applied to remove outliers and high-frequency noise. Then we employ autocorrelation to calculate the periodicity for both the amplitude and phase of signal ratio [18]. The signal with better periodicity between amplitude and phase is selected to extract the respiration rate using Fast Fourier Transform (FFT) [19].

\footnotetext{
${ }^{8}$ The sampling rate of USRP is $800 \mathrm{kHz}$. We reduce the sampling rate to $1 \mathrm{kHz}$ to process data quickly.
} 
The output of $\operatorname{argmax}(\operatorname{abs}(\mathrm{fft}(\mathrm{x})))$ corresponds to the dominant frequency, which is the respiration rate of the target. We employ a respiration monitor belt logger sensor NUL-236 [8] to provide ground-truths as shown in Figure 16. The ground-truth of respiration rate is calculated as $\frac{\text { number of breaths }}{\text { recordingtime }}$ and the unit is Beats Per Minute (BPM). We develop a GUI to visualize the signal variations in real time, as shown in Figure 17.

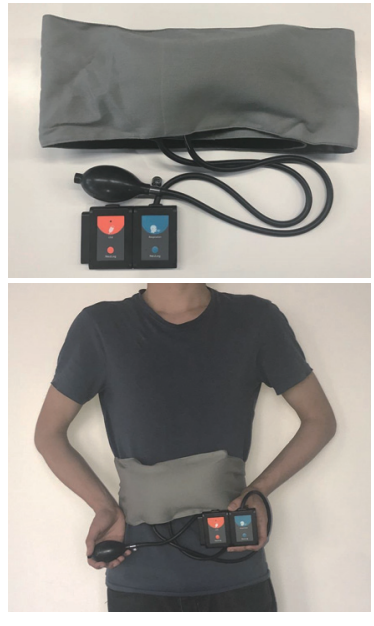

Fig. 16. Belt logger sensor.

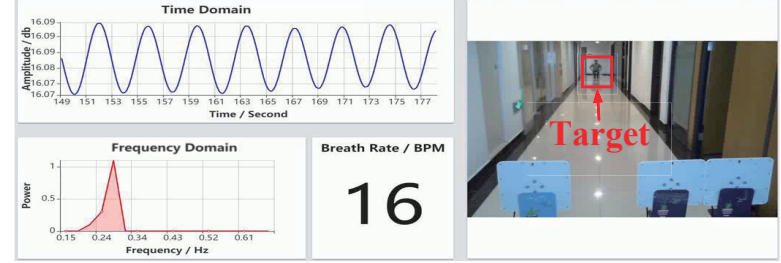

(a) Respiration sensing at $25 \mathrm{~m}$ distance

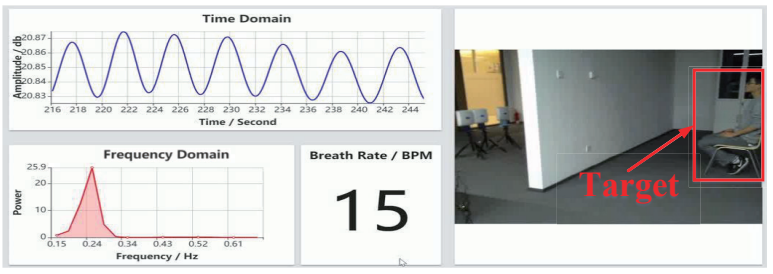

(b) Respiration sensing through wall

Fig. 17. Web-based user interface for LoRa-based respiration sensing.

4.1.2 Effectiveness of Respiration Sensing. Existing contactless respiration sensing systems usually require one of the transceivers to be close to the target, which may not be feasible in certain application scenarios (e.g., disaster rescue). Using LoRa, we can place both transceivers far away from the target and behind walls. We evaluate the sensing accuracy with varying distances and through walls.

Long-range sensing: We recruit five participants and ask each of them to sit in a chair and breathe naturally. We vary the distance between the human target and the transceivers from $5 \mathrm{~m}$ to $25 \mathrm{~m}$ with a step size of $5 \mathrm{~m}$. We record the respiration data for each participant. Figure 18a plots the amplitude variation of signal ratio at a distance of $25 \mathrm{~m}$. The increase and decrease in the amplitude correspond to the inhalation and exhalation during the respiration process. We can obtain the repiration rate of the target, which is $0.25 \times 60=15 \mathrm{BPM}$ (Breaths Per Minute) as shown in Figure 18b. Figure 19 plots mean absolute error of respiration rate as a function of distance from $5 \mathrm{~m}$ to $25 \mathrm{~m}$. The results show that the mean absolute error is $0.1 \mathrm{BPM}$ at 5 meters and slightly increases to $0.37 \mathrm{BPM}$ when the subject is 25 meters away from the transceivers. Therefore, our system can reliably sense human respiration rate even when the subject is $25 \mathrm{~m}$ away from the transceivers.

Through-wall sensing: We further study the sensing performance by including walls between the transceiver pair and the target. As shown in Figure 17b, the target sits in a room while the LoRa transceivers are placed behind the wall. We repeat the respiration sensing process and increase the distance from $5 \mathrm{~m}$ to $15 \mathrm{~m}$. We find that when there is one wall in between, we can still sense respiration at a distance of $15 \mathrm{~m}$ and the mean absolute error is as low as 0.18BPM (17.39BPM vs. 17.57BPM). We also explore the sensing limit by including two walls. When the target and transceivers are separated with 2 walls at a distance of $15 \mathrm{~m}$, the mean absolute error is still less than 0.2BPM. These results indicate that our system can reliably monitor human respiration even in the challenging scenario with one or two walls between the transceivers and the target. 


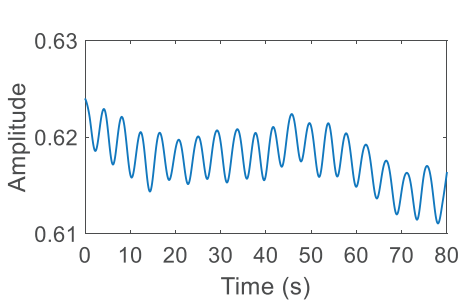

(a) Amplitude of signal ratio

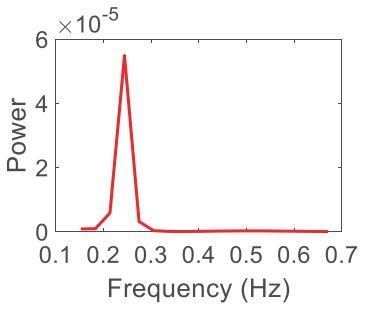

(b) Frequency domain

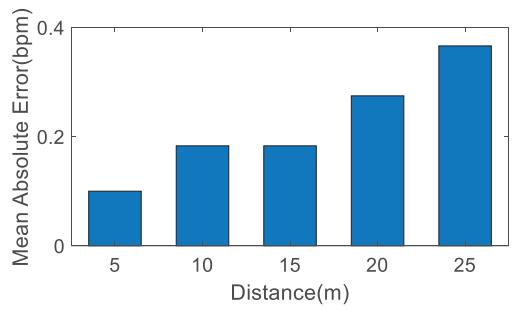

Fig. 18. Signals for respiration sensing at $25 \mathrm{~m}$.

Fig. 19. The mean absolute error of respiration rate versus distance to the transceivers.

4.1.3 Performance under Different Affecting Factors. We further evaluate the performance of our respiration sensing system with varying spacing between transceivers, different human targets, and various indoor environments.

Different LoS distance: We vary the spacing between the transceivers from $1 \mathrm{~m}$ to $5 \mathrm{~m}$ at a step size of $1 \mathrm{~m}$. The target is 10 meters away from the transceivers with a wall between them. We observe that human respiration can be well sensed for all the LoS distances. As shown in Figure 20, the mean absolute error is only 0.23BPM.

Different human targets: To demonstrate the effect of user diversity, five participants including one female and four male students are recruited for evaluation. We record five sets of respiration monitoring data for each participant at $10 \mathrm{~m}$ away with one wall between the target and sensing device. Figure 21 shows the acquired average respiration rates of the five participants and the corresponding ground-truth obtained by the belt logger sensor. The overall estimation accuracy is as high as $98.8 \%$.

Different indoor environments: We also evaluate our respiration sensing system in three practical environments: home, office, and meeting room, as shown in Figure 23. In the three different environments, the target is asked to sit in a chair behind a wall. With different room layouts, the distances from the target to the transceivers in home, office, and meeting room are $7.5 \mathrm{~m}, 6.6 \mathrm{~m}$ and $10 \mathrm{~m}$ with one wall in between, respectively. We observe clear fluctuations and cycles, matching the respiration rate. In Figure 22, the results show that the mean absolute errors for three different environments with different users are 0.18BPM, 0.17BPM and 0.17BPM, respectively. The tables and other furniture in front of the target has no effect on the respiration sensing performance. The LoRa signal can penetrate the wall as well as these furniture. The highly accurate results obtained in all environments demonstrate the robustness of long-range and through-wall respiration sensing using LoRa.

4.1.4 Interference from Surroundings. Here, we investigate the impact of interference from surroundings on respiration sensing. We ask another person to walk across between the target and transceivers, and we find that the signal is affected only briefly and the abnormal signal variations quickly revert back to normal periodic cycles. As shown in Figure. 24, when the other person is not inside the coverage area of the directional antenna,

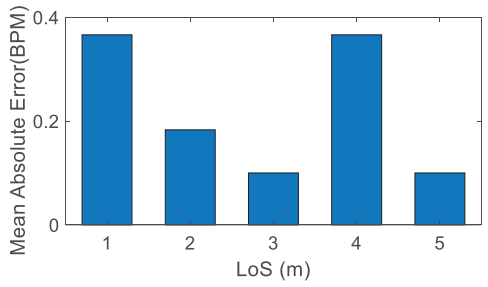

Fig. 20. Impact of LoS distance.
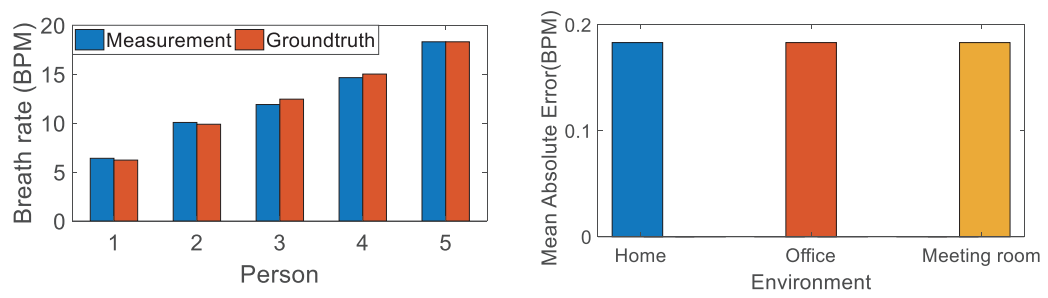

Fig. 21. Impact of different human targets. Fig. 22. Impact of different environments. 


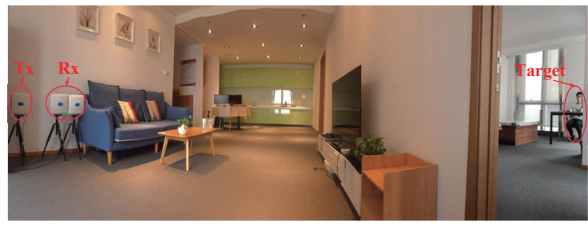

(a) Home

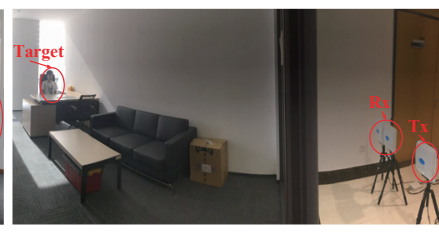

(b) Office

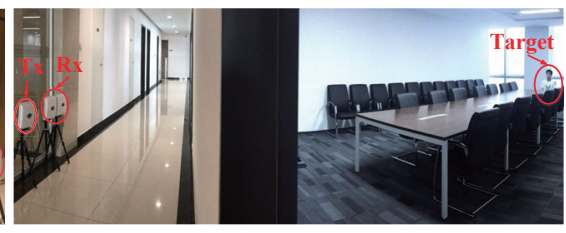

(c) Meeting room

Fig. 23. Three different experimental environments for respiration sensing.

even when the other person is quite close to the target, the interference is small enough to be ignored. On the other hand, when the other person is inside the sensing area, even when the other person is $10 \mathrm{~m}$ away from the target, the interference still exists. Moreover, we test the case of blocking the target and transceiver pair with a moving board. We observe that when the board is moving, the respiration sensing is interfered. However, once the moving board becomes stationary, the respiration information can be accurately sensed without any problem. This means that blockage is not an issue but object movement brings in interference.

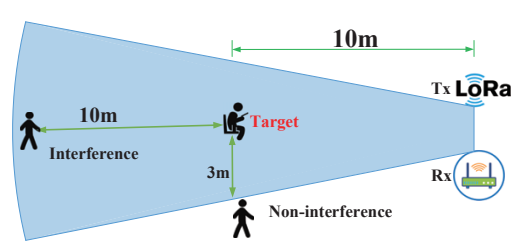

Fig. 24. Respiration sensing: Interference from surroundings.

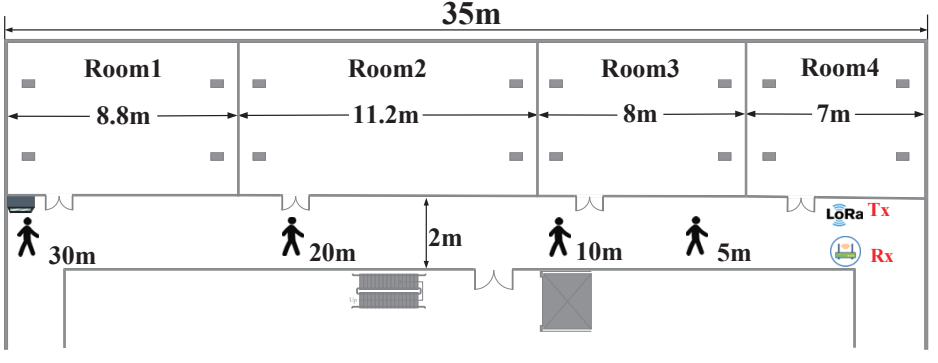

Fig. 25. Human tracking: Deployment scenario.

4.1.5 Comparison with Different Sensing Technologies and Systems. We compare the proposed LoRa sensing system with the state-of-the-art methods based on Wi-Fi, FMCW and Ultrasound. We implement three stateof-the-art sensing systems based on Wi-Fi [62], FMCW radar [5] and Ultrasound [55] and compare them with the proposed LoRa sensing system in three aspects: 1) sensing range; 2) through-wall sensing capability; and 3) accuracy.

Experiment setup: As shown in Fig. 26a, we implement the WiFi-based system proposed in [62] with one Wi-Fi transceiver pair. Each transceiver is a Gigabyte mini-PC equipped with an Intel $5300 \mathrm{Wi}$-Fi card. The signal is transmitted in the $5.24 \mathrm{GHz}$ frequency band. The transmitter/receiver are placed with a distance of $1 \mathrm{~m}$ in between at the same height. The packet transmission rate is 20 packets per second.

In Fig. 26b, we implement the FMCW-based system proposed in [5] with SGR Semiconductors' $24 \mathrm{GHz}$ radar chipset RKA1203. The chipset can generate linear chirps with a 1024us chirp period and sweep across $1 \mathrm{GHz}$ bandwidth starting from $23.5 \mathrm{GHz}$. The radar is equipped with one transmission antenna and two receiving antennas. This $24 \mathrm{GHz}$ radar is relatively cheap $(\$ 100)$ and we notice that the expensive radar device with more advanced hardware can achieve better sensing performance in terms of sensing range. However, the price is much higher than LoRa and Wi-Fi hardware.

In Fig. 26c, we implement the Ultrasound-based sensing system proposed in [55]. We employ a laptop (Dell Precision 5520 with Intel Xeon CPU E3-1505M, 16GB RAM) and connect it to a pair of commodity speaker (JBL Jembe, $6 \mathrm{Watt}$ ) and microphone (SAMSON MeteorMic), serving as the audio transceivers. 


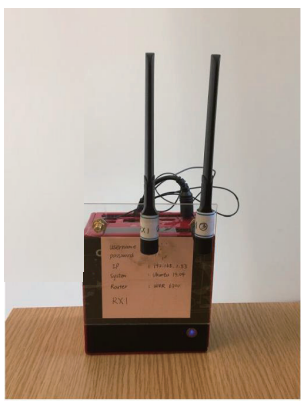

(a) WiFi device.

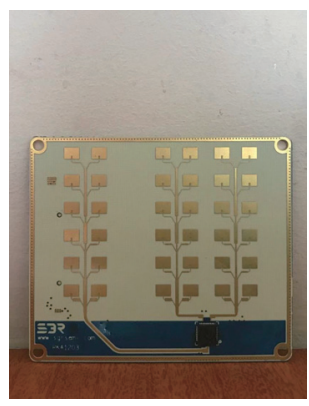

(b) FMCW Radar.

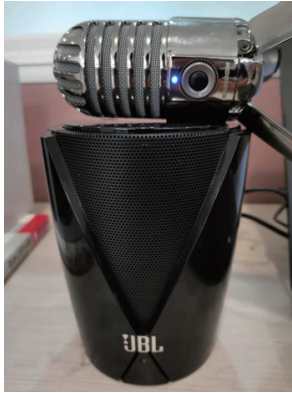

(c) Ultrasound device.

Fig. 26. Experiment devices

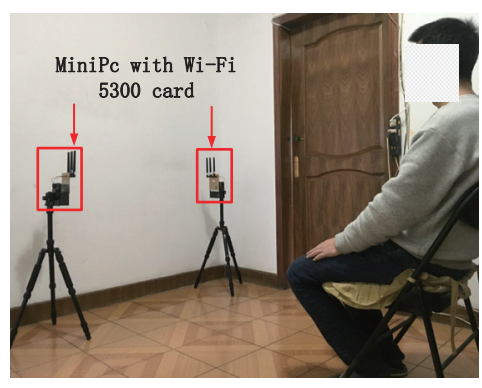

(a) WiFi scenario.

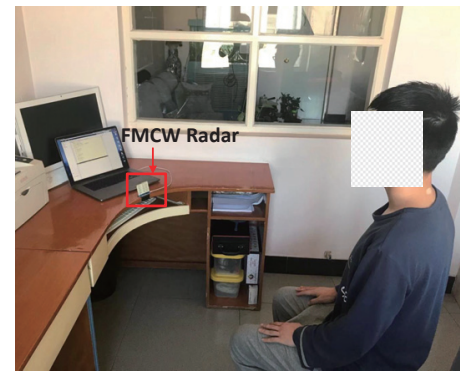

(b) FMCW Radar scenario.

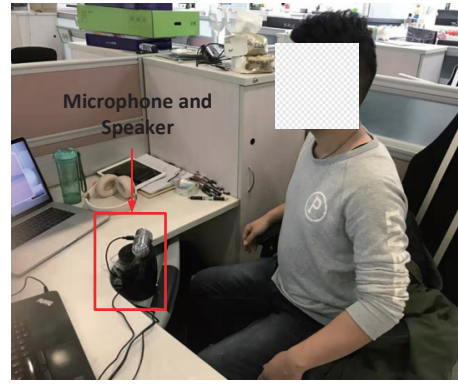

(c) Ultrasound scenario.

Fig. 27. Experiment scenarios

We deploy all the devices in the indoor environment as shown in Fig. 27. We first conduct experiments to measure the sensing range (maximum working distance between the target and the sensing device) for each wireless technology. Without loss of generality, we also take human respiration detection as the example application to compare the sensing performance. The sensing ranges for WiFi, FMCW radar and Ultrasound are $4 \mathrm{~m}, 2 \mathrm{~m}$ and $0.6 \mathrm{~m}$, respectively as shown in Fig. 28a. Compared with these sensing methods, our LoRa-based sensing system can achieve a much larger $25 \mathrm{~m}$ sensing range.

To evaluate the through-wall capability, the target sits in a room and we place the sensing device in another room with different number of walls between the target and sensing device. We find that LoRa system can still reliably monitor human respiration even in the challenging scenario when there are two walls between the sensing device and the target and the distance between the target and sensing device is $15 \mathrm{~m}$. For Wi-Fi sensing system, it works when one of the transceivers is placed in another room with one wall in between. When both Wi-Fi transceivers are located in a different room, it has difficulty to sense the human respiration. For FMCW radar and Ultrasound devices, the sensing systems fail to detect human respiration through one wall. We want to point out that expensive high-end FMCW radar with directional horn antenna array and higher transmit power has through-wall capability to sense human respiration. However, the price is expensive (a few thousands dollars) and it is unfair to compare it with the cheap commodity LoRa and Wi-Fi devices. Thus, we choose this 


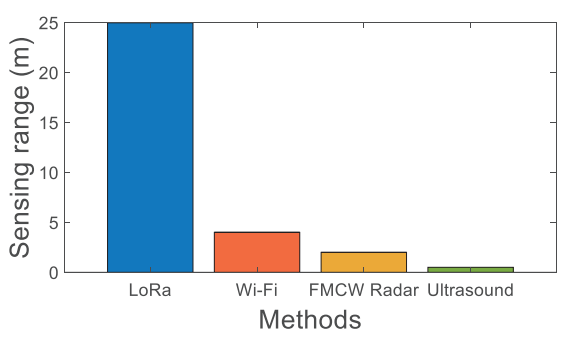

(a) Sensing range.

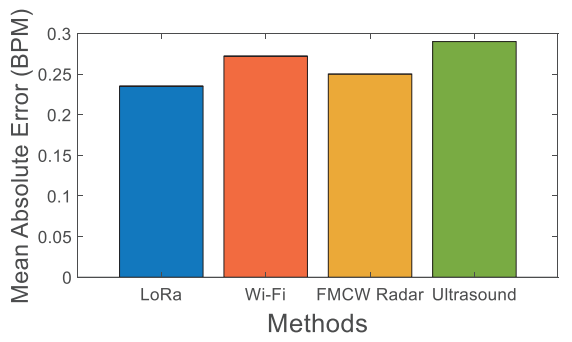

(b) Accuracy.

Fig. 28. Comparison between different sensing methods.

SOC (system on chip) radar which has a comparable price with other sensing devices. Moreover, the reason that Ultrasound can not go through walls is the stronger attenuation than electromagnetic waves during the process of penetrating through walls.

Fig. 28b shows the detection accuracy of different sensing systems. We observe that the mean absolute error (MAE) of LoRa is $0.23 \mathrm{BPM}$ even when the target is far away $(25 \mathrm{~m})$ from the LoRa sensing device. For the other three systems (Wi-Fi, FMCW radar, Ultrasound), the MAE at their largest sensing range are 0.27 BPM, 0.25 BPM and 0.29 BPM, respectively. We conclude that these methods have comparable accuracy. However, the sensing range is much larger and through-wall capability is much stronger for LoRa.

\subsection{Long-distance Through-wall Human Tracking}

In this subsection, we present the design and implementation of a real-time human tracking system in a long corridor. As shown in Figure 25, there are four rooms along the corridor and the total length is $35 \mathrm{~m}$. We only deploy a pair of transceivers at one end of the corridor. Our system is able to track human walking in this long corridor. Also we evaluate the accuracy of walking distance and direction at different positions.

4.2.1 System Implementation. To track human walking distance and direction, we first segment the walking activity from the time-series of signal ratio, and further determine the starting and ending time of the motion. Note that the variance of signal ratio amplitude is very sensitive to target movements. Thus the state transition of the amplitude variance can be used to identify the starting and ending time of walking. We utilize a $0.7 \mathrm{~s}$ sliding window (with a step size of $0.35 \mathrm{~s}$ ) to estimate the amplitude variance. Then we select 1.5 times of the maximum variance as the threshold to detect the motion and determine whether there is human walking in the corridor. After segmenting the signal of walking activity, we calculate the vectors on the tangential direction. The phase difference of vectors on the tangential direction is unwrapped to calculate the phase change, which is then used to derive the walking distance. We identify the phase rotation direction (clockwise or counter-clockwise) to determine whether the target is moving towards or away from the transceivers. If the phase change is positive, the target is moving towards the transceivers; if the phase change is negative, then the target is moving away. As shown in Figure 29, we developed a GUI to visualize human walking in real time .

4.2.2 Overall Performance. We recruit five participants and each participant walks continuously, traversing the paths between all four rooms. As shown in Figure 30, we plot the amplitude of signal ratio while a target walks between different rooms (e.g., Room $1 \rightarrow$ Room $2 \rightarrow$ Room $1 \rightarrow$ Room3). The motion state can be clearly identified. Based on the walking distance and direction, our system can achieve $100 \%$ accuracy in monitoring human walking from one room to another, as well as differentiating walking directions (e.g., Room1 $\rightarrow$ Room2 or Room2 $\rightarrow$ Room1). 


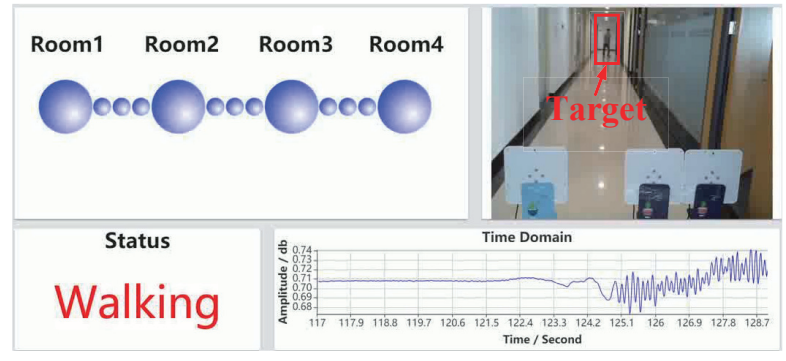

(a) Walking status in a long corridor

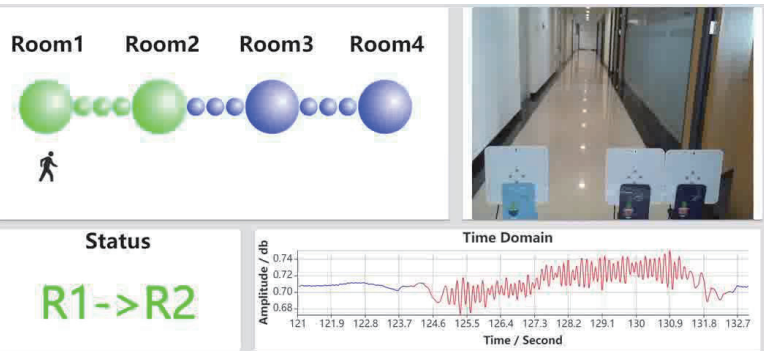

(b) Room tracking

Fig. 29. Web-based user interface for LoRa-based human tracking.

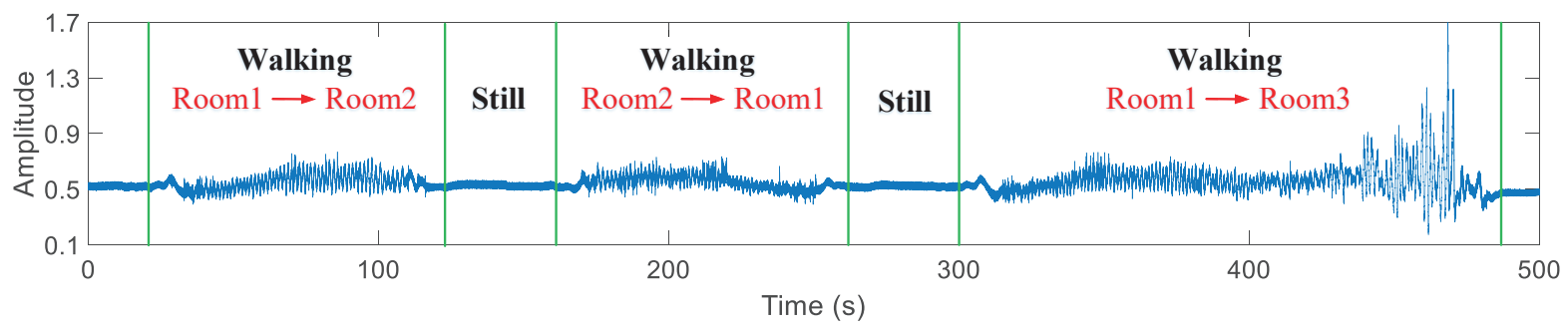

Fig. 30. Signal for walking between rooms.

4.2.3 Walking Distance and Direction Estimation. To evaluate the accuracy of human tracking, we conduct experiments with both real human target and dummy. We include the experiment with dummy because the dummy's movement can be precisely controlled (millimeter-level precision) with the help of a sliding track. On the other hand, the human's movement is complicated and the moving speed is not exactly a constant. Thus, with the dummy, we can eliminate the unavoidable interference and focus on the part we would like to evaluate. Thus, for a comprehensive evaluation, we include both real human and dummy as target in our experiments.

We conduct experiments with dummies and real people in the same environment and the experiment setups are also kept the same. As shown in Fig. 31, the distance between the dummy/person and the sensing device is varied from $10 \mathrm{~m}$ to $30 \mathrm{~m}$ at a step size of $5 \mathrm{~m}$. We move the dummy for $1 \mathrm{~m}$ on the track or ask the person to move for $1 \mathrm{~m}$. The experiments are repeated 10 times at each location. The moving distance can be calculated by accurately measuring the amount of phase rotation of the signal. Fig. 32 shows the displacement estimation errors at different distances. The average displacement error with dummies is $3.8 \mathrm{~cm}$. The average displacement error with real human targets is $6.8 \mathrm{~cm}$. Low errors can be achieved with both dummies and real human targets. We observe that the error variance with human is slightly larger than that with dummies. We think the slightly higher errors with real human are mainly due to two reasons: (1) It is difficult to precisely control the starting and ending positions of human walking. (2) When human targets walk, the involuntary body movements and non-constant speed make the ground truth measurements biased. Furthermore, we found that due to the large reflection area, the body reflection signal dominates among the reflected signals. Thus, even though other bodypart movements such as arm swinging also reflect signal which interferes the walking tracking, the body-reflected signal is much stronger and the effect of interference is limited. 


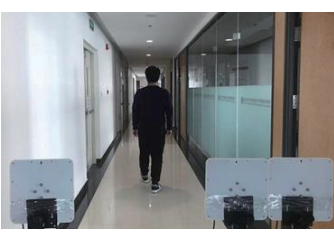

(a) Real person walking.

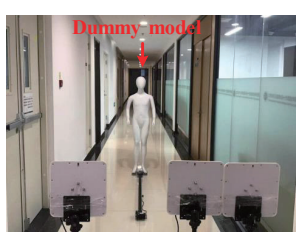

(b) Dummy model moving

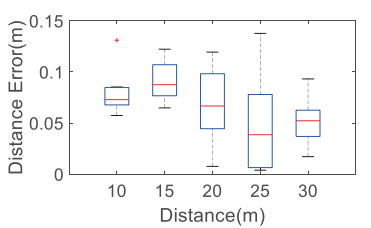

(a) Real person results.

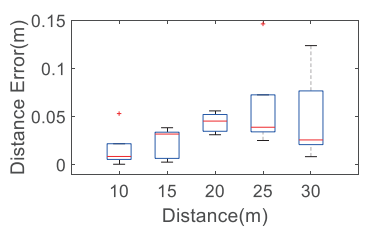

(b) Dummy results.

Fig. 31. Experiment scenarios

Next we investigate whether the target moving direction can be identified instantly once the target starts walking. We conduct experiments with different number of signal samples to perform the walking direction estimation. For all test users, our system can reliably detect the walking direction at $100 \%$ accuracy with a latency of 0.7 seconds. This demonstrates that our system can accurately track human walking from far away.

4.2.4 Through-wall Human Tracking. We also validate the sensing performance when there are one or two walls between the target and the sensing devices. The dummy is moved $1 \mathrm{~m}$ along the perpendicular bisector of the transceivers $15 \mathrm{~m}$ away. The average distance estimation error is $4.27 \mathrm{~cm}$. When the target and transceivers are separated by $15 \mathrm{~m}$ with 2 walls in between, the average absolute error is $4.79 \mathrm{~cm}$, which demonstrates the high accuracy of LoRa's through-wall tracking capability. The walking orientation can be obtained from phase rotation direction (clockwise and counterclockwise), which can be reliably detected with $100 \%$ accuracy. We want to point out that we only employ one transceiver pair in this experiment. With multiple receivers, we expect to obtain richer and finer-grained information via LoRa sensing.

\section{RELATED WORK}

LoRa-based communication and sensing. Prior work on LoRa network mainly focuses on enabling IoT applications with LoRa's long-distance communication capability. Tahera et al. utilize LoRa link to transfer human activity data monitored by sensors [22] [21]. LoRaMote is attached to a person's arm with a band, which is equipped with a set of sensors (e.g., temperature and pressure) to monitor the human target's health condition [42]. Ke et al. deploy LoRa nodes on windows, corridors, and roofs to form a mesh network to transfer sensor-collected data for campus monitoring [28]. Philip et al. collect industrial sensor data using LoRa in the workshop [47]. All these works only exploit the long-range communication capability of LoRa. Little attention has been paid to leveraging the LoRa signal itself as an effective sensing tool. Islam et al. study the feasibility of LoRa in indoor localization, which utilizes the received signal strength indicator (RSSI) measurement and validate that LoRa is more stable for localization than both WiFi and Bluetooth Low Energy (BLE) [23]. Although effective in localization, we note that the RSSI measurement is still a coarse information, which fails to sense fine-grained human activities. The recent work proposed by Rajalakshmi et al. develops a multi-band backscatter system for localization with sub-centimeter-sized devices [36]. In contrast, in this work, we aim to explore the passive sensing capability of LoRa signal with off-the-shelf LoRa hardware without using dedicated sensors.

Other wireless sensing techniques. Other wireless technologies widely employed for sensing include millimeter wave radar [58], Doppler radar [38], UWB radar [51], FMCW radar [13][64], commodity WiFi [12], RFID [54] and Zigbee [40]. Using the special-purpose device such as FMCW radar and UWB radar, Adib et al. track users' breathing and the sensing accuracy is quite high, where users might be 8 meters away from the device [13]. However, these dedicated sensing devices are quite costly. Among all the commodity device based solutions, such as WiFi, RFID, and Zigbee, WiFi based passive human sensing is the most popular one and has found many interesting applications, ranging from localization [31] to human respiration detection [63] and 
gesture recognition [37]. For example, Zhang et al. leverage commodity Wi-Fi devices to detect human respiration, which could be 2-4 meters away from each other [63] [62]. IndoTrack employs Wi-Fi devices to achieve human tracking within the range of less than 6 meters [32]. However, the long-range through-wall passive sensing capability is still missing in existing commodity wireless sensing technologies. In this regard, LoRa presents new opportunities in enabling long-range and through-wall passive sensing with commodity devices under very low power consumption.

\section{LIMITATION AND DISCUSSION}

In this work, we focus on utilizing LoRa signal to achieve long-range contactless sensing. We briefly discuss the limitation and future direction of our work below.

\subsection{Deployment Issue}

The fact that wireless sensing obtains information from the reflected signals makes the sensing range much smaller compared to the signal communication range. All wireless signals have the same issue. For example, the communication range of WiFi is $20 \sim 50$ meters but its sensing range is only 3 6 meters, whereas RFID and mmWave-based systems show an even smaller sensing range of 1 3 meters. The LoRa gateway deployment in the outdoor environment can be sparse due to its long communication range. We want to point out that, the LoRa indoor gateway deployment can be denser [59][27]. Also each LoRa gateway is expected to be connected to quite a few LoRa nodes. For LoRa sensing to work, we do not require both the gateway and the node to be close to the target $(10-20 \mathrm{~m})$. As long as one of the nodes is close to the target, LoRa signal can be applied for sensing. In the future work, we plan to explore LoRa sensing in outdoor environment with a sparser deployment. We believe there is still a space to further increase the sensing range. We expect to achieve around $50-100 \mathrm{~m}$ sensing range with advanced signal processing in outdoor environment.

\subsection{Privacy Issue}

Note that potential privacy issues may be raised with long-range sensing. However, compared with existing video-based monitoring, the privacy concern is less. We believe anti-sensing is one interesting solution to address the concern. Interfering signal can be employed to confuse the receiver. The target-reflected signal and the interfering signal will be mixed together and make sensing the target difficult.

\subsection{Multi-target Sensing}

Multi-target sensing is a well-known challenge and may be the biggest challenge in contactless wireless sensing. Two methods have been proposed recently. The first one is to employ independent component correlation (ICA) [61] method to separate signals reflected from multiple targets. This method requires to know the number of targets. Furthermore, multiple antennas are needed at the receiver and the signals need to be linearly combined at each antenna. The number of observed signal sources also needs to be larger than the number of targets. The other method [57] proposes to employ information from multiple dimensions (space, time and frequency) to separate signals for $\mathrm{WiFi}$-based sensing. Compared to ICA, the requirement is relaxed but still multiple antennas are needed. A good news is that Semtech is planning to equip LoRa gateways with multiple antennas (4-8) [6][20] and we are optimistic these methods can be applied to enable multi-target LoRa sensing.

\section{CONCLUSION}

This work explores the sensing model, techniques and applications of LoRa - a new LPWANs technology designed for the Internet of Things - to achieve long-range through-wall human sensing. This paper tries to answer several fundamental questions such as "What is the underlining model and theory for LoRa sensing?"; "What is the 
sensing range limit of LoRa?" and so on. In answering these questions, we first derive the sensing model with LoRa signals and propose to utilize the signal ratio of two antennas to increase the sensing range significantly. We further reveal the relationship between the target moving distance/orientation and the resultant phase change, and study the factors affecting the sensing performance. Extensive experiments in a variety of environments show that (1) Subtle human respiration can be sensed when the target is 15 meters away with one wall in between; (2) Human walking distance and orientation can be tracked even when the target is 30 meters away; and (3) LoRa offers significant sensing range improvement over existing commodity wireless technologies such as WiFi and RFID. We believe the proposed system would enable a large range of new applications.

\section{ACKNOWLEDGMENTS}

This work is supported by the National Natural Science Foundation of China (No. 61802373), the Youth Innovation Promotion Association, Chinese Academy of Sciences (No. 2020109), the National Key Research and Development Plan (No. 2016YFB1001200), the EU CHIST-ERA RadioSense Project, the EU Horizon 2020 research and innovation programme IDEA-FAST (No. 853981), the Google European Doctoral Fellowship in Wireless Networking.

\section{A APPENDIX}

Here we present the detailed derivation procedure of the received signal in Equation 3. Suppose that a LoRa node transmits the signal $T x(t)=e^{j \pi k t^{2}+j 2 \pi f_{c} t}$, if a signal arrives at the receiver through $N$ different paths, and the $n^{t h}$ path has a propagation delay of $\tau_{n}(t)$, the received signal can then be represented as:

$$
\begin{aligned}
R x(t) & =\sum_{n=1}^{N} a_{n}(t) T x\left(t-\tau_{n}(t)\right)=\sum_{n=1}^{N} a_{n}(t) e^{j \pi k\left(t-\tau_{n}(t)\right)^{2}+j 2 \pi f_{c}\left(t-\tau_{n}(t)\right)} \\
& =e^{j \pi k t^{2}+j 2 \pi f_{c} t} \sum_{n=1}^{N} a_{n}(t) e^{-j 2 \pi f_{c} \tau_{n}(t)-j 2 \pi k t \tau_{n}(t)+j \pi k \tau_{n}(t)^{2}} \\
& =e^{j \pi k t^{2}+j 2 \pi f_{c} t} \sum_{n=1}^{N} a_{n}(t) e^{-j 2 \pi\left(k \tau_{n}(t) t+f_{c} \tau_{n}(t)-k \frac{\tau_{n}(t)^{2}}{2}\right)} \\
& =T x(t) \cdot \sum_{n=1}^{N} H_{n}(t)
\end{aligned}
$$

where $H_{n}(t)=a_{n}(t) e^{-j 2 \pi\left(k \tau_{n}(t) t+f_{c} \tau_{n}(t)-k \frac{\tau_{n}(t)^{2}}{2}\right)}$ is the impulse response of the $n^{t h}$ path, $a_{n}(t)$ is the complex valued representation of attenuation and initial phase offset of the $n^{\text {th }}$ path. Note that in long-range LoRa sensing, the transceiver spacing is much smaller than the distance between the target and transceivers, thus the transmitter and receiver can be considered as collocated and $\tau_{n}(t)$ is then calculated as: $\tau_{n}(t)=\frac{2 D(t)}{c}$, where $D(t)$ is the distance between transceivers and target, and $c$ is the speed of light. Therefore, $H_{n}(t)$ can be rewritten as:

$$
H_{n}(t)=a_{n}(t) e^{-j 4 \pi\left(\frac{k D(t)}{c} t+f_{c} \frac{D(t)}{c}-\frac{k D(t)^{2}}{c^{2}}\right)}
$$

Note that the speed of light $c$ is $3 \times 10^{8} \mathrm{~m} / \mathrm{s}$, thus the term with $\frac{1}{c^{2}}$ is very small and can be ignored. Thus, $H_{n}(t)$ can be simplified as: $H_{n}(t)=a_{n}(t) e^{-j 4 \pi\left(\frac{k D(t)}{c} t+\frac{f_{c} D(t)}{c}\right)}$. For chirp-based lora signal, each chirp has a period of $T$ and a time window of $t$ may contain multiple chirps. Thus $H_{n}(t)$ can be further represented as: $H_{n}(t)=$ $a_{n}(t) e^{-j 4 \pi\left(\frac{k D(t)}{c}(t \% T)+\frac{f_{c} D(t)}{c}\right)}$. The phase of signal contains two parts: $\phi_{1}=4 \pi \frac{k D(t)}{c}(t \% T)$ and $\phi_{2}=4 \pi \frac{f_{c} D(t)}{c}$. For the first part, because the bandwidth of LoRa signal is small (i.e., $B=125 \mathrm{kHz}$ ), in one sweep period of the chirp signal, the phase change of this item is $4 \pi \frac{B D(t)}{c}$ because $k=B / T$. This phase change is a very small value (three 
orders of magnitude smaller than $2 \pi$ ). The first phase part thus leads to a high frequency component $1 / T$ on signal (e.g., if $T=10 \mathrm{~ms}, 1 / T=100 \mathrm{~Hz}$ ). For the second part $\phi_{2}, D(t)$ can be written as: $D(t)=D_{0}+v t$, where $D_{0}$ is the initial distance of the target and $v$ is the speed of the target. This part leads to a low frequency component, which is $\frac{1}{2 \pi} \frac{\delta \phi_{2}}{\delta t}=\frac{2 f_{c} v}{c}$ (e.g., for $f_{c}=915 \mathrm{MHz}, v=1 \mathrm{~m} / \mathrm{s}, \frac{2 f_{c} v}{c}=6 \mathrm{~Hz}$ ). The frequencies of the two parts differ by two orders of magnitude, we can thus employ a low-pass filter to remove the high frequency component and only keep the low frequency component which is related to target movements. Hence, we can further simplify the impulse response as $H_{n}(t)=a_{n}(t) e^{-j 2 \pi \frac{f_{c} 2 D(t)}{c}}=a_{n}(t) e^{-j 2 \pi f_{c} \tau_{n}(t)}$, and the received signal as follows.

$$
R x(t)=e^{j \pi k t^{2}+j 2 \pi f_{c} t} \sum_{n=1}^{N} a_{n}(t) e^{-j 2 \pi f_{c} \tau_{n}(t)}
$$

\section{REFERENCES}

[1] 2006. GNURadio. https://www.gnuradio.org/.

[2] 2013. USRP B210. https://www.ettus.com/all-products/UB210-KIT/.

[3] 2015. Arduino. https://store.arduino.cc/usa/arduino-uno-rev3.

[4] 2015. The LoRa alliance. https://www.lora-alliance.org/.

[5] 2016. FMCW radar. http://www.sgrsemi.com/?lg=en.

[6] 2016. LoRaGuide. https://developer.orange.com/wp-content/uploads/LoRa-Device-Developer-Guide-Orange.pdf.

[7] 2016. Semtech SX1276 Transceiver. https://www.semtech.com/products/wireless-rf/lora-transceivers/sx1276.

[8] 2017. Respiration Monitor Belt logger sensor NUL-236. https://neulog.com/respiration-monitor-belt/.

[9] 2018. Alibaba Cloud aims to connect 10 billion devices by 2023. https://technode.com/2018/03/28/alibaba-cloud-iot/.

[10] 2018. Semtech and Comcast's machineQ Announce LoRaWAN Network Availability in 10 Cities. https://investors.semtech.com/newsreleases/news-release-details/semtech-and-comcasts-machineq-announce-lorawan-network.

[11] Fadel Adib, Zachary Kabelac, Dina Katabi, and Rob Miller. 2014. WiTrack: motion tracking via radio reflections off the body. In Proc. of NSDI.

[12] Fadel Adib and Dina Katabi. 2013. See through walls with WiFi!. In ACM SIGCOMM 2013 Conference. ACM, 75-86.

[13] Fadel Adib, Hongzi Mao, Zachary Kabelac, Dina Katabi, and Robert C Miller. 2015. Smart homes that monitor breathing and heart rate. In Proceedings of the 33rd annual ACM conference on human factors in computing systems. ACM, 837-846.

[14] Kamran Ali, Alex X Liu, Wei Wang, and Muhammad Shahzad. 2017. Recognizing keystrokes using WiFi devices. IEEE fournal on Selected Areas in Communications 35, 5 (2017), 1175-1190.

[15] Thomas Ameloot, Patrick Van Torre, and Hendrik Rogier. 2018. LoRa indoor performance: an office environment case study. In ACES2018, the International Applied Computational Electromagnetics Society Symposium. 1-2.

[16] Aloÿs Augustin, Jiazi Yi, Thomas Clausen, and William Townsley. 2016. A study of LoRa: Long range \& low power networks for the internet of things. Sensors 16, 9 (2016), 1466.

[17] Albert Berni and WO Gregg. 1973. On the utility of chirp modulation for digital signaling. IEEE Transactions on Communications 21,6 (1973), 748-751.

[18] George EP Box, Gwilym M Jenkins, Gregory C Reinsel, and Greta M Ljung. 2015. Time series analysis: forecasting and control. John Wiley \& Sons.

[19] E Oran Brigham and E Oran Brigham. 1988. The fast Fourier transform and its applications. Vol. 448. prentice Hall Englewood Cliffs, NJ.

[20] Arliones Hoeller, Richard Demo Souza, Onel L Alcaraz López, Hirley Alves, Mario de Noronha Neto, and Glauber Brante. 2018. Analysis and Performance Optimization of LoRa Networks With Time and Antenna Diversity. IEEE Access 6 (2018), 32820-32829.

[21] Tahera Hossain, Yusuke Doi, Tahia Tazin, Md Atiqur Rahman Ahad, and Sozo Inoue. 2018. Activity Recognition by Using LoRaWAN Sensor. In Proceedings of the 2018 ACM International foint Conference on Pervasive and Ubiquitous Computing. ACM, 58-61.

[22] Tahera Hossain, Yusuke Doi, Tahia Tazin, Md Atiqur Rahman Ahad, and Sozo Inoue. 2018. Study of LoRaWAN Technology for Activity Recognition. In Proceedings of the 2018 ACM International foint Conference on Pervasive and Ubiquitous Computing. ACM, 1449-1453.

[23] Bashima Islam, Md Tamzeed Islam, and Shahriar Nirjon. 2017. Feasibility of LoRa for Indoor Localization. on-line, from semanticscholar. org (2017), 1-11.

[24] Brian K Jennison. 2000. Performance of a linear frequency-modulated signal detection algorithm. In Record of the IEEE 2000 International Radar Conference. IEEE, 447-450.

[25] Te-Yu Jason Kao and Jenshan Lin. 2013. Vital sign detection using 60-GHz Doppler radar system. In 2013 IEEE International Wireless Symposium (IWS). IEEE, 1-4.

[26] Fadel Adib Zachary Kabelac Dina Katabi. 2015. Multi-Person Localization via RF Body Reflections. (2015). 
[27] Kai-Hsiang Ke, Qi-Wen Liang, Guan-Jie Zeng, Jun-Han Lin, and Huang-Chen Lee. 2017. A LoRa Wireless Mesh Networking Module for Campus-Scale Monitoring. In Proceedings of the 16th ACM/IEEE International Conference on Information Processing in Sensor Networks. Association for Computing Machinery, 259-260.

[28] Kai-Hsiang Ke, Qi-Wen Liang, Guan-Jie Zeng, Jun-Han Lin, and Huang-Chen Lee. 2017. A LoRa wireless mesh networking module for campus-scale monitoring: demo abstract. In Proceedings of the 16th ACM/IEEE International Conference on Information Processing in Sensor Networks, IPSN. ACM, 259-260.

[29] Shengjie Li, Zhaopeng Liu, Yue Zhang, Xiaopeng Niu, Leye Wang, and Daqing Zhang. 2019. A real-time and robust intrusion detection system with commodity Wi-Fi. In UbiComp/ISWC Adjunct. ACM.

[30] Tianxing Li, Qiang Liu, and Xia Zhou. 2016. Practical human sensing in the light. In Proceedings of the 14th Annual International Conference on Mobile Systems, Applications, and Services. ACM, 71-84.

[31] Xiang Li, Shengjie Li, Daqing Zhang, Jie Xiong, Yasha Wang, and Hong Mei. 2016. Dynamic-music: accurate device-free indoor localization. In Proceedings of the 2016 ACM International foint Conference on Pervasive and Ubiquitous Computing. ACM, 196-207.

[32] Xiang Li, Daqing Zhang, Qin Lv, Jie Xiong, Shengjie Li, Yue Zhang, and Hong Mei. 2017. IndoTrack: Device-Free Indoor Human Tracking with Commodity Wi-Fi. Proceedings of the ACM on Interactive, Mobile, Wearable and Ubiquitous Technologies 1, 3 (2017), 72.

[33] Sujuan Liu, Chuyu Xia, and Zhenzhen Zhao. 2016. A low-power real-time air quality monitoring system using lpwan based on lora. In 2016 13th IEEE International Conference on Solid-State and Integrated Circuit Technology (ICSICT). IEEE, 379-381.

[34] Samarth Mathur, Anand Sankar, Prajwal Prasan, and B Iannucci. 2017. Energy Analysis of LoRaWAN Technology for Traffic Sensing Applications. In Proceedings of the Intelligent Transportation Society of America (ITS) World Congress, Montréal, QC, Canada, Vol. 29.

[35] Christopher A. Miles. 2007. Through-the-Wall Surveillance: A New Technology for Saving Livess. National Institute of Justice (2007).

[36] Rajalakshmi Nandakumar, Vikram Iyer, and Shyamnath Gollakota. 2018. 3D Localization for Sub-Centimeter Sized Devices. In Proceedings of the 16th ACM Conference on Embedded Networked Sensor Systems. ACM, 108-119.

[37] Rajalakshmi Nandakumar, Bryce Kellogg, and Shyamnath Gollakota. 2014. Wi-fi gesture recognition on existing devices. arXiv preprint arXiv:1411.5394 (2014).

[38] M Nowogrodzki, DD Mawhinney, and HF Milgazo. 1984. Non-invasive microwave instruments for the measurement of respiration and heart rates. NAECON 1984 (1984), 958-960.

[39] Alain Olivier, Guillermo Bielsa, Irene Tejado, Michele Zorzi, Joerg Widmer, and Paolo Casari. 2016. Lightweight indoor localization for 60-GHz millimeter wave systems. In 2016 13th Annual IEEE International Conference on Sensing, Communication, and Networking (SECON). IEEE, 1-9.

[40] Neal Patwari, Lara Brewer, Quinn Tate, Ossi Kaltiokallio, and Maurizio Bocca. 2014. Breathfinding: A wireless network that monitors and locates breathing in a home. IEEE Journal of Selected Topics in Signal Processing 8, 1 (2014), 30-42.

[41] Yao Peng, Longfei Shangguan, Yue Hu, Yujie Qian, Xianshang Lin, Xiaojiang Chen, Dingyi Fang, and Kyle Jamieson. 2018. PLoRa: a passive long-range data network from ambient LoRa transmissions. In Proceedings of the 2018 Conference of the ACM Special Interest Group on Data Communication. ACM, 147-160.

[42] Juha Petäjäjärvi, Konstantin Mikhaylov, Matti Hämäläinen, and Jari linatti. 2016. Evaluation of LoRa LPWAN technology for remote health and wellbeing monitoring. In 2016 10th International Symposium on Medical Information and Communication Technology (ISMICT). IEEE, $1-5$.

[43] Juha Petäjäjärvi, Konstantin Mikhaylov, Rumana Yasmin, Matti Hämäläinen, and Jari Iinatti. 2017. Evaluation of LoRa LPWAN technology for indoor remote health and wellbeing monitoring. International fournal of Wireless Information Networks 24, 2 (2017), 153-165.

[44] Qifan Pu, Sidhant Gupta, Shyamnath Gollakota, and Shwetak Patel. 2013. Whole-home gesture recognition using wireless signals. In Proceedings of the 19th annual international conference on Mobile computing \& networking. ACM, 27-38.

[45] Wenjie Ruan, Lina Yao, Quan Z Sheng, Nickolas JG Falkner, and Xue Li. 2014. Tagtrack: Device-free localization and tracking using passive rfid tags. In Proceedings of the 11th international conference on mobile and ubiquitous systems: computing, networking and services. ICST (Institute for Computer Sciences, Social-Informatics and ąy, 80-89.

[46] Ronald W Schafer. 2011. What is a Savitzky-Golay filter?[lecture notes]. IEEE Signal Processing Magazine 4 (2011), 111-117.

[47] Philipp Sommer, Yannick Maret, and Dacfey Dzung. 2018. Low-Power Wide-Area Networks for Industrial Sensing Applications. In 2018 IEEE International Conference on Industrial Internet (ICII). IEEE, 23-32.

[48] Vamsi Talla, Mehrdad Hessar, Bryce Kellogg, Ali Najafi, Joshua R Smith, and Shyamnath Gollakota. 2017. Lora backscatter: Enabling the vision of ubiquitous connectivity. Proceedings of the ACM on Interactive, Mobile, Wearable and Ubiquitous Technologies 1, 3 (2017), 105.

[49] Zhao Tian Andrew T. Campbell Xia Zhou Tianxing Li, Chuankai An. 2015. Human Sensing Using Visible Light Communication. In Proceedings of the 21th Annual International Conference on Mobile Computing and Networking. ACM, 331-344.

[50] Teodor Tomic, Korbinian Schmid, Philipp Lutz, Andreas Domel, Michael Kassecker, Elmar Mair, Iris Lynne Grixa, Felix Ruess, Michael Suppa, and Darius Burschka. 2012. Toward a fully autonomous uav: Research platform for indoor and outdoor urban search and rescue. IEEE robotics and automation magazine 19, 3 (2012).

[51] Swaroop Venkatesh, Christopher R Anderson, Natalia V Rivera, and R Michael Buehrer. 2005. Implementation and analysis of respirationrate estimation using impulse-based UWB. In MILCOM 2005-2005 IEEE Military Communications Conference. IEEE, 3314-3320.

Proc. ACM Interact. Mob. Wearable Ubiquitous Technol., Vol. 4, No. 2, Article 68. Publication date: June 2020. 
[52] Anran Wang and Shyamnath Gollakota. 2019. MilliSonic: Pushing the Limits of Acoustic Motion Tracking. (2019).

[53] Ju Wang, Hongbo Jiang, Jie Xiong, Kyle Jamieson, Xiaojiang Chen, Dingyi Fang, and Binbin Xie. 2016. LiFS: low human-effort, device-free localization with fine-grained subcarrier information. In Proceedings of the 22nd Annual International Conference on Mobile Computing and Networking. ACM, 243-256.

[54] Ju Wang, Jie Xiong, Hongbo Jiang, Xiaojiang Chen, and Dingyi Fang. 2017. D-watch: embracing ąřbadąś multipaths for device-free localization with cots RFID devices. IEEE/ACM Transactions on Networking (TON) 25, 6 (2017), 3559-3572.

[55] Tianben Wang, Daqing Zhang, Yuanqing Zheng, Tao Gu, Xingshe Zhou, and Bernadette Dorizzi. 2018. C-FMCW based contactless respiration detection using acoustic signal. Proceedings of the ACM on Interactive, Mobile, Wearable and Ubiquitous Technologies 1,4 (2018), 170.

[56] Yanwen Wang and Yuanqing Zheng. 2019. TagBreathe: Monitor Breathing with Commodity RFID Systems. IEEE Transactions on Mobile Computing (2019).

[57] Yaxiong Xie, Jie Xiong, Mo Li, and Kyle Jamieson. 2018. mD-Track: Leveraging Multi-Dimensionality in Passive Indoor Wi-Fi Tracking. The 25th Annual International Conference on Mobile Computing and Networking (MobiCom) (2018).

[58] Zhicheng Yang, Parth H Pathak, Yunze Zeng, Xixi Liran, and Prasant Mohapatra. 2016. Monitoring vital signs using millimeter wave. In Proceedings of the 17th ACM International Symposium on Mobile Ad Hoc Networking and Computing. ACM, 211-220.

[59] Rumana Yasmin, Juha Petajajarvi, Konstantin Mikhaylov, and Ari Pouttu. 2018. Large and Dense LoRaWAN Deployment to Monitor Real Estate Conditions and Utilization Rate. In 2018 IEEE 29th Annual International Symposium on Personal, Indoor and Mobile Radio Communications (PIMRC).

[60] N.J. Young. 1984. Linear fractional transformations in rings and modules. Linear Algebra Appl. 56 (1984), 251 - 290. https://doi.org/10. 1016/0024-3795(84)90131-9

[61] Shichao Yue, Hao He, Hao Wang, Hariharan Rahul, and Dina Katabi. 2018. Extracting Multi-Person Respiration from Entangled RF Signals. ACM International foint Conference on Pervasive and Ubiquitous Computing (Ubicomp 2018) (2018).

[62] Youwei Zeng, Dan Wu, Ruiyang Gao, Tao Gu, and Daqing Zhang. 2018. FullBreathe: Full Human Respiration Detection Exploiting Complementarity of CSI Phase and Amplitude of WiFi Signals. Proceedings of the ACM on Interactive, Mobile, Wearable and Ubiquitous Technologies 2, 3 (2018), 148.

[63] Fusang Zhang, Daqing Zhang, Jie Xiong, Hao Wang, Kai Niu, Beihong Jin, and Yuxiang Wang. 2018. From fresnel diffraction model to fine-grained human respiration sensing with commodity wi-fi devices. Proceedings of the ACM on Interactive, Mobile, Wearable and Ubiquitous Technologies 2, 1 (2018), 53.

[64] Mingmin Zhao, Shichao Yue, Dina Katabi, Tommi S Jaakkola, and Matt T Bianchi. 2017. Learning sleep stages from radio signals: A conditional adversarial architecture. In Proceedings of the 34th International Conference on Machine Learning-Volume 70. JMLR. org, $4100-4109$. 